\title{
On the Variational Equilibrium as a Refinement of the Generalized Nash Equilibrium
}

\author{
Ankur A. Kulkarni $\quad$ Uday V. Shanbhag*
}

\begin{abstract}
We are concerned with a class of Nash games in which the players' strategy sets are coupled by a shared constraint. A widely employed solution concept for such games, referred to as generalized Nash games, is the generalized Nash equilibrium (GNE). The variational equilibrium (VE) [6] is a specific kind of GNE given by a solution of the variational inequality formed from the common constraint and the mapping of the gradients of player objectives. Our contribution is a theory that provides sufficient conditions for ensuring that the existence of a GNE implies the existence of a VE; in such an instance, the VE is said to be a refinement of the GNE. For certain games our conditions are shown to be necessary. This theory rests on a result showing that, in both the primal and the primal-dual space, the GNE and the VE are equivalent upto the Brouwer degree of two suitably defined functions, whose zeros are the GNE and VE, respectively. The refinement of the GNE is of relevance to pure, applied and computational game theory. Our results unify some previously known facts pertaining to such equilibria and are utilized in showing that shared-constraint Nash-Cournot games arising in power markets do indeed admit a refinement.
\end{abstract}

\section{Introduction}

This paper concerns noncooperative $N$-player generalized Nash games [12] (or coupled constrained games [25]) where players are assumed to have continuous strategy sets that are dependent on the strategies of their adversaries. Such games represent generalizations of classical noncooperative games that have traditionally allowed for strategic interactions between players to be expressed only through their objective functions. In a frequently encountered class of generalized Nash games, player strategies are required to satisfy a common coupling constraint. These games are called generalized Nash games with shared constraints [25] and are the focus of this paper.

Let $\mathcal{N}=\{1,2, \ldots, N\}$ be a set of players, $m_{1}, \ldots, m_{N}$ be positive integers and $m=\sum_{i=1}^{N} m_{i}$. For each $i \in \mathcal{N}$, let $U_{i} \subseteq \mathbb{R}^{m_{i}}$ represent player $i$ 's strategy set, $x_{i} \in U_{i}$ be his strategy and $\varphi_{i}: \mathbb{R}^{m} \rightarrow \mathbb{R}$ be his objective function. We use the following notation: by $x$ we denote the tuple $\left(x_{1}, x_{2}, \ldots, x_{N}\right)$, $x^{-i}$ denotes the tuple $\left(x_{1}, \ldots, x_{i-1}, x_{i+1}, \ldots, x_{N}\right)$ and $\left(y_{i}, x^{-i}\right)$ the tuple $\left(x_{1}, \ldots, x_{i-1}, y_{i}, x_{i+1}, \ldots, x_{N}\right)$. A shared constraint is a requirement that the tuple $x$ be constrained to lie in a set $\mathbb{C} \subseteq \mathbb{R}^{m}$. In the generalized Nash game with shared constraint $\mathbb{C}$, player $i$ is assumed to solve the parameterized optimization problem,

$$
\begin{array}{|lll|}
\hline \mathrm{A}_{i}\left(x^{-i}\right) & \begin{array}{l}
\text { minimize } \\
x_{i} \\
\text { subject to }
\end{array} & \varphi_{i}\left(x_{i} ; x^{-i}\right) \\
&
\end{array}
$$

${ }^{*}$ Both authors are at the Department of Industrial and Enterprise Systems Engineering, University of Illinois, UrbanaChampaign. IL 61801, U.S.A., and are reachable at (akulkar3, udaybag@illinois.edu). This work was supported by NSF grant CCF-0728863. The authors would like to thank Prof. P. Tseng and Prof. J-S. Pang for their advice and comments on an earlier draft of this paper. 
where for each $i \in \mathcal{N}$ the set-valued maps $K_{i}: \prod_{j \neq i} \mathbb{R}^{m_{j}} \rightarrow 2^{\mathbb{R}^{m_{i}}}$ and the map $K: \mathbb{R}^{m} \rightarrow 2^{\mathbb{R}^{m}}$, are defined as

$$
K_{i}\left(x^{-i}\right):=\left\{y_{i} \in \mathbb{R}^{m_{i}} \mid\left(y_{i}, x^{-i}\right) \in \mathbb{C}\right\}, \quad \forall i \in \mathcal{N} \text { and } \quad K(x):=\prod_{i \in \mathcal{N}} K_{i}\left(x^{-i}\right) \quad \forall x \in \mathbb{R}^{m} .
$$

For simplicity, we have dropped the sets $U_{i}$ in the above optimization problems and have assumed that $\mathbb{C}$ is contained in $\prod_{i \in \mathcal{N}} U_{i}$. We denote the resulting game resulting from the above optimization problems by $\mathcal{G}$. The solution concept applied to analyze such games is called the generalized Nash equilibrium (GNE).

Definition 1.1 (Generalized Nash equilibrium (GNE)) A strategy tuple $x \equiv\left(x_{1}, x_{2}, \ldots, x_{N}\right)$ is a generalized Nash equilibrium of $\mathcal{G}$ if $x_{i} \in S O L\left(A_{i}\left(x^{-i}\right)\right)$ for all $i \in \mathcal{N}$.

Here $\operatorname{SOL}(P)$ refers to the solution set of an optimization problem $P$. The GNE is an extension of the social equilibrium proposed by Debreu [4]; see also [25, 1] and the recent survey [6] for more on this. We now introduce another solution concept. The variational equilibrium (VE) is a specific kind of GNE defined in $[6,8]$ :

Definition 1.2 (Variational equilibrium (VE)) A strategy tuple $x$ is said to be a variational equilibrium of $\mathcal{G}$ if $x$ is a solution of $V I(\mathbb{C}, F)$.

The notation $\operatorname{VI}(\mathbb{C}, F)$ denotes a variational inequality with mapping $F$ and a set $\mathbb{C}$ (see Section 1.1 ), where $F: \mathbb{R}^{m} \rightarrow \mathbb{R}^{m}$ is the function given by

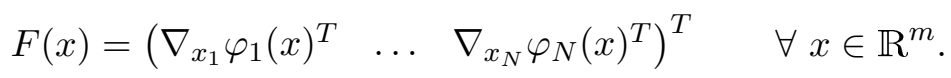

The goal of this paper is to provide a theoretical framework for developing sufficiency conditions for the VE to be a refinement of the GNE. From an economic standpoint, the notion of refinement of an equilibrium is rooted in the belief that the concept of an equilibrium may be far too weak to serve as a solution concept. Therefore, for a stronger solution concept, one avenue lies in refining the equilibrium concept in games. Given such a motivation, what properties are of relevance in constructing such a refinement? If the weakness of the original concept is on the count that certain equilibria have less economic justification, then a refinement should formalize this by excluding such equilibria. Naturally, for a refinement to be useful, it should lead to a nonempty set of equilibria when the original solution concept admits equilibria. Thus, a refinement of the set of equilibria of a game is (a) a subset satisfying a certain rule, where this rule has the property that (b) any game with a nonempty set of equilibria also possesses an equilibrium satisfying this rule. Both the refined equilibria and the rule generating them are collectively referred to as the refinement. Refinements of equilibria are considered in detail by Myerson [19] and have been previously sought for a host of solution concepts in both static and dynamic games [3]. For instance, the subgame-perfect Nash equilibrium is a refinement of the Nash equilibrium of a dynamic game (see [20, ch. 3.8]); trembling hand perfect [26] and proper [18] equilibria are refinements of mixed Nash equilibria in static finite strategy games [3, 27].

It is known from [5] that every VE is a GNE. Thus this paper focuses on showing that, under suitable conditions, the existence of a GNE implies the existence of a VE, i.e. (b). There are at least two motivations for studying this question which we describe below. First, for a modeler (studying, say, traffic flow or bandwidth allocation), equilibria of a game can be regarded as "outcomes" that would result if the game were to be played out in practice and when a game has many equilibria there is no clear indication of this outcome. GNEs of games such as $\mathcal{G}$ have properties that, we believe, warrant a refinement. These games are known to admit a large number, and in some cases, a manifold of GNEs 
(see [6]; also Theorem 20 in Appendix A.1). In fact, in the following example, every strategy tuple in $\mathbb{C}$ is a GNE.

Example 1. Game where every strategy tuple is a GNE: Consider a game where player $i$ has real valued strategies and solves

\begin{tabular}{|lll|}
\hline $\mathrm{A}_{i}\left(x^{-i}\right)$ & $\begin{array}{c}\text { minimize } \\
x_{i} \\
\text { subject to }\end{array} \quad X=\alpha \quad: \lambda_{i}$, \\
\end{tabular}

where $X=\sum_{i \in \mathcal{N}} x_{i}$. Games such as this arise commonly in network routing. The Karush-Kuhn-Tucker (KKT) conditions characterizing the GNE, $x^{*}$, of this game are

$$
\left(x_{i}^{*} \ell\left(X^{*}\right)\right)^{\prime}=\lambda_{i}, \quad \forall i \in \mathcal{N} \quad \text { and } \quad X^{*}=\alpha .
$$

Clearly, every point in the set $\mathbb{C}=\{x \mid X=\alpha\}$ is a GNE of this game. Does a subset of these characterize economically justifiable strategic behavior?

Another shortcoming of the GNE is that there are settings for which not every GNE is meaningful from an real-world standpoint. The first motivation for our study is to present a refinement of the GNE that will retain a set of GNEs that is smaller, yet economically meaningful, even under these settings. We argue below that it is indeed the VE that has this property. Consider a game like in the above example and suppose that the Lagrange multipliers can be interpreted as prices charged on the players by an administrator for whom the players are anonymous. The VE is also known to be the GNE with the same Lagrange multipliers corresponding to the shared constraint [5]. Thus for this game VE has the additional property of being an equilibrium with uniform prices whereas the GNE corresponds to one with discriminatory prices. Since players are anonymous, and hence indistinguishable from each other, it is unreasonable to assume that the administrator can charge discriminatory prices and the only equilibria that make sense are ones in which the same price is charged to all players, i.e. the VE. If the VE is indeed a refinement of the GNE, then the VE exists whenever the GNE does and thus may be used as a solution concept in lieu of the GNE.

Our second motivation arises from the need to characterize and compute GNEs. Consider a game in which the $i^{\text {th }}$ player solves the parameterized convex program

\begin{tabular}{|c|c|c|}
\hline $\mathrm{A}_{i}\left(x^{-i}\right)$ & $\begin{array}{l}\underset{x_{i}}{\operatorname{minimize}} \\
\text { subject to }\end{array}$ & $\begin{array}{l}\varphi_{i}\left(x_{i} ; x^{-i}\right) \\
A x \geq b \quad\left(\lambda_{i}\right), \\
\quad x \geq 0\end{array}$ \\
\hline
\end{tabular}

where $\varphi_{i}\left(x_{i} ; x^{-i}\right)$ is convex in $x_{i}$ for all $x^{-i}$ and $\lambda_{i}$ is the player-specific Lagrange multiplier corresponding to the shared-constraint. Then the equilibrium conditions of the game are given by the rank-deficient complementarity problem

$$
\begin{array}{ll}
0 \leq x_{i} \perp \nabla_{x_{i}} f_{i}-A_{i}^{T} \lambda_{i} \geq 0, & \forall i \in \mathcal{N}, \\
0 \leq \lambda_{i} \perp A x-b \geq 0, & \forall i \in \mathcal{N},
\end{array}
$$

suggesting that the original equilibrium problem is ill-posed. A simple step for making the game well-posed requires that the players have consistent multipliers, denoted by say $\lambda$, and the resulting equilibrium conditions are given by the following square complementarity problem:

$$
\begin{array}{cc}
0 \leq x_{i} \perp \nabla_{x_{i}} f_{i}-A_{i}^{T} \lambda \geq 0, & \forall i \in \mathcal{N}, \\
0 \leq \lambda \perp A x \geq b, & \forall i \in \mathcal{N} .
\end{array}
$$


In general, obtaining a GNE requires a solution of this ill-posed system which leads to a quasi-variational inequality in the primal-space and a non-square complementarity problem in the primal-dual space. The $\mathrm{VE}$, on the other hand, requires the solution of either a variational inequality (primal space) or a square complementarity problem (primal-dual space) both of which being far more tractable objects.

This has several implications both from an analytical and a computational standpoint. In particular, in attempting to analyzing GNEs, one may choose to focus primarily on VEs. Such a direction is sensible only when it is known that the the existence of a GNE is sufficient for the existence of a VE. Furthermore, as regards computation, it has been common practice $[6,22,16]$ to compute the VE instead of the GNE and limit computation only to the class of games, $\mathcal{S}_{2}$, for which the VE exists. Notice that $\mathcal{S}_{2}$ is contained in the class of games, $\mathcal{S}$, for which the VE is a refinement of the GNE. But sufficiency conditions for the solvability of variational inequalities (such as those in [7]) that may be applied for checking if a game belongs to $\mathcal{S}_{2}$ do not exploit the existence of a GNE to show a solution to $\mathrm{VI}(\mathbb{C}, F)$; these theorems apply to a class, $\mathcal{S}_{2}^{\prime}$, smaller than $\mathcal{S}_{2}$, for which one can claim the existence of a VE independently of knowledge of the existence of a GNE. This in turn has limited the practice of computation of GNEs only to games in $\mathcal{S}_{2}^{\prime}$. Identification of $\mathcal{S}$ leads to an identification of $\mathcal{S}_{2}$, rather than the smaller set $\mathcal{S}_{2}^{\prime}$.

The contribution of this paper is a theory that gives sufficient conditions for a game to belong to $\mathcal{S}$. For certain classes of games, these conditions are also seen to be necessary. Also, $\mathcal{S}$ is shown to contain classes other than $\mathcal{S}_{2}^{\prime}$ in itself. Ours is perhaps the first work on the refinement of equilibria in the context of generalized Nash games. Our sufficient conditions are expressed in terms of the Brouwer degree, which is seen to relate the GNE and the VE in a profound manner.

In both, primal and primal-dual space, we show that there are functions $v$ and $g$ whose zeros are VEs and GNEs respectively, such that the Brouwer degrees of $v$ and $g$, with respect to zero are equal. This paves the way for identifying subclasses of $\mathcal{S}$. In the primal setting, we show the above result with $v$ and $g$ taken as the natural maps of the quasi-variational inequality (whose solutions capture all GNEs) and the variational inequality (whose solutions are the VEs). Through a novel equation reformulation of the primal-dual GNE, this degree theoretic approach is extended to the primal-dual space. Finally, we show that these sufficiency conditions can be applied on an instance of a shared-constraint Nash-Cournot game arising in power markets.

The paper is organized as follows. Sections 2 and 3 deal with the treatment of the refinement question in primal and primal-dual spaces, respectively. Section 4 contains some examples of shared constraint games and describes how the sufficiency conditions for a game to admit the VE as a refinement may be applied to shared-constraint Nash-Cournot game arising in power markets. We conclude with some final considerations in Section 5. Before proceeding, we outline our assumptions and provide some technical background.

\section{$1.1 \quad$ Background}

We make the following assumptions throughout the paper.

Assumption 1 For each $i \in \mathcal{N}$, the objective function $\varphi_{i} \in C^{2}$ and $\varphi_{i}\left(x_{i} ; x^{-i}\right)$ is convex in $x_{i}$ for all $x^{-i}$. Unless otherwise mentioned, $\mathbb{C}$ is closed, convex and has a nonempty interior.

A brief background on variational inequalities and Brouwer degree theory follows.

Recall problems $\mathrm{A}_{i}$ from Section 1. Under assumption $1, x_{i}$ is optimal for $\mathrm{A}_{i}\left(x^{-i}\right)$ if and only if $\nabla_{i} \varphi_{i}(x)^{T}\left(y_{i}-x_{i}\right) \geq 0$, for all $y_{i} \in K_{i}\left(x^{-i}\right)$, where $K_{i}$ is as defined in (1). Thus if $x=\left(x_{1}, \ldots, x_{N}\right)$ is a GNE of $\mathcal{G}$ if and only if it solves the quasi-variational inequality (QVI) [7] below.
Find $x \in K(x)$
such that
$F(x)^{T}(y-x) \geq 0$
$\forall y \in K(x)$
$(\mathrm{QVI}(K, F))$ 
For the closed convex set $\mathbb{C}$ and function $F$, the variational inequality $\operatorname{VI}(\mathbb{C}, F)$ is the following problem, a solution of which was defined to be the VE in Definition 1.2.

$$
\text { Find } x \in \mathbb{C} \quad \text { such that } \quad F(x)^{T}(y-x) \geq 0 \quad \forall y \in \mathbb{C} \text {. }
$$

The natural map of $\operatorname{VI}(\mathbb{C}, F), \mathbf{F}_{\mathbb{C}}^{\text {nat }}: \mathbb{R}^{m} \rightarrow \mathbb{R}^{m}$, defined as $\mathbf{F}_{\mathbb{C}}^{\text {nat }}(v)=v-\Pi_{\mathbb{C}}(v-F(v))$ where $\Pi_{\mathbb{C}}$ : $\mathbb{R}^{m} \rightarrow \mathbb{C}$ is the Euclidean projection on $\mathbb{C}$, provides an equation reformulation of the VI. Let $\operatorname{dom}(K):=$ $\{v \mid K(v) \neq \emptyset\}$ and $\widetilde{\mathbf{F}}_{K}^{\text {nat }}: \operatorname{dom}(K) \rightarrow \mathbb{R}^{m}$ denote a similar natural map for $\operatorname{QVI}(K, F)$ defined as $\widetilde{\mathbf{F}}_{K}^{\text {nat }}(v):=v-\Pi_{K(v)}(v-F(v))$, for $v \in \operatorname{dom}(K)$. We then have:

Proposition $1([7])$ A vector $v$ solves $V I(\mathbb{C}, F)$ if and only if $\mathbf{F}_{C}^{\text {nat }} b b(v)=0$ and $v$ solves $Q V I(K, F)$ if and only if $\widetilde{\mathbf{F}}_{K}^{n a t}(v)=0$.

We frequently use the following result on projections on closed convex sets.

Lemma 2 ([7]) Let $V \subseteq \mathbb{R}^{m}$ be a closed convex set and $x$ be a point in $\mathbb{R}^{m}$. Then the projection of $x$ on $D, \Pi_{V}(x)$, satisfies $\left(y-\Pi_{V}(x)\right)^{T}\left(\Pi_{V}(x)-x\right) \geq 0$ for each $y$ in $V$.

$\mathbf{F}_{V}^{\text {nat }}$ is a continuous function when $V$ is closed and convex but the continuity of $\widetilde{\mathbf{F}}_{K}^{\text {nat }}$ relies on the continuity of the set-valued map $K$ (see [2]).

Lemma 3 (Lemma 2.8.2[7]) Let $x \in \operatorname{dom}(K)$ and $y$ be any point in $\mathbb{R}^{m}$. Then $\phi(x, y):=\mathbf{F}_{K(x)}^{n a t}(y)$ is continuous at $(x, y)$ for all $y \in \mathbb{R}^{m}$ if and only if $K(\cdot)$ is continuous at $x$.

Proposition 4.7.1 in [7, page 401] provides sufficient conditions for $K$ to be continuous when $\mathbb{C}$ is given by an algebraic constraint.

The Brouwer degree $[15,21,9]$ of a function is a topological concept that allows us to claim the existence of zeros of the function in a specified open set. Degree theory has been previously applied to the study of variational inequalities $[10,11,7]$. Let $\Omega \subset \mathbb{R}^{m}$ be an open bounded set, $f: \bar{\Omega} \rightarrow \mathbb{R}^{m}$ be continuous and $p \in \mathbb{R}^{m} \backslash f(\partial \Omega)$. We say the Brouwer degree of $f$ with respect to $p$ on $\Omega$, denoted as $\operatorname{deg}(f, \Omega, p)$, is well defined if $p \notin f(\partial \Omega)$ and it exists only for such $p$. Let $\mathbf{1}: \mathbb{R}^{m} \rightarrow \mathbb{R}^{m}$ denote the identity map. $\operatorname{deg}(f, \Omega, p)$ is an integer with the following properties.

1. (Normalization $) \operatorname{deg}(\mathbf{1}, \Omega, p)=1$ if and only if $p \in \Omega$.

2. (Solvability) $\operatorname{deg}(f, \Omega, p) \neq 0$ then $f(x)=p$ for some $x \in \Omega$.

3. (Homotopy invariance) $\operatorname{deg}(H(\cdot, t), \Omega, p)$ is independent of $t \in[0,1]$ for any continuous function $H: \bar{\Omega} \times[0,1] \rightarrow \mathbb{R}^{m}$ and $p \in \mathbb{R}^{m}$ such that $p \notin \cup_{t \in[0,1]} H(\partial \Omega, t) . H$ is called a homotopy.

4. (Translation invariance) $\operatorname{deg}(f-p, \Omega, 0)=\operatorname{deg}(f, \Omega, p)$

5. (Degree of injective maps) Let $f$ be continuous and injective and $f(x)=p$ for some $x \in \Omega$. Then $\operatorname{deg}(f, \Omega, p)= \pm 1$.

Note that the converse of property 2 is not true in general. i.e. if $f(x)=p$ for some $x$ in $\Omega$ and $\operatorname{deg}(f, \Omega, p)$ is well defined, it does not imply that the degree not zero. But if $f$ is continuous and injective, such a claim can be made, cf. property 5 . 


\section{Primal Generalized Nash and Variational Equilibria}

In this section we begin the development of our theory of the refinement of the GNE. Our analysis is restricted to the primal space and does not impose any algebraic form on $\mathbb{C}$, relying mainly on geometric and convex analytic arguments. A primal-dual analysis that uses an algebraic form for $\mathbb{C}$ and a primal-dual characterization of the GNE and VE is included in Section 3. The material pertaining specifically to the refinement of the GNE is encompassed in Section 2.2. The following material upto the end of Section 2.1 establishes some preliminary results useful for the results in Section 2.2 and for a better understanding of $\mathrm{QVI}(K, F)$. We begin by recalling that every VE is a GNE, a result shown by Facchinei et al. [5, Theorem 2.1].

Theorem 4 For any continuous function $\Gamma: \mathbb{R}^{m} \rightarrow \mathbb{R}^{m}$, if $x$ is a solution of $V I(\mathbb{C}, \Gamma)$ then $x$ is a solution of $Q V I(K, \Gamma)$.

The next set of results help develop a deeper understanding of the set-valued map $K$.

\subsection{The properties of $K$}

Fig 1(a) shows a convex set $\mathbb{C}$ and $K(x)$ for an $x \in \mathbb{R}^{2}$, assuming $m_{1}=m_{2}=1$ and $N=2$. Notice that $K(x)$ is formed as a product, namely $K_{1}\left(x_{2}\right) \times K_{2}\left(x_{1}\right)$. In general $\operatorname{dom}(K):=\{x \mid K(x) \neq \emptyset\}$ is not $\mathbb{R}^{m}$ and there may be points outside $\mathbb{C}$ whose image under at least one of the $K_{i}$ 's is empty. For instance in Fig $1(\mathrm{a})$, notice the point $y=\left(y_{1}, y_{2}\right)$ for which both $K_{1}\left(y_{2}\right)$ and $K_{2}\left(y_{1}\right)$ are empty. The

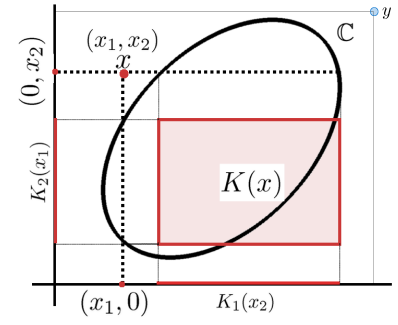

(a) $x$ and $K(x)$ for the set $\mathbb{C}$ and $y$ such that $K(y)=\emptyset$
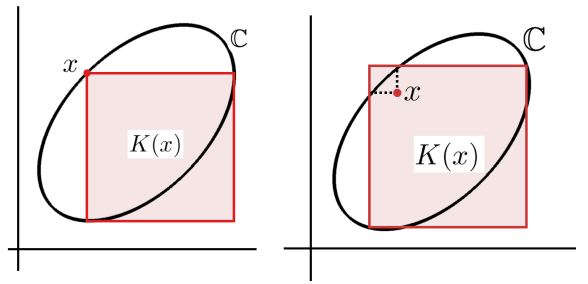

(b) $x$ on the boundary of $\mathbb{C}$ and in the interior of $\mathbb{C}$

following Lemma mentions some more relationships between $K$ and $\mathbb{C}$. See Appendix A.2 for proof.

Lemma 5 Let $\mathbb{C}$ be a closed set in $\mathbb{R}^{m}$ and $K$ be as given in (1). Then the following hold:

1. If $\mathbb{C}=\prod_{i \in \mathcal{N}} \mathbb{C}_{i}$, where $\mathbb{C}_{i} \subseteq \mathbb{R}^{m_{i}}$ for every $i \in \mathcal{N}$, are nonempty, not necessarily convex sets, then $K(x)=\mathbb{C}$ for every $x$ in $\mathbb{C}$ and is empty otherwise.

2. For any $\mathbb{C}$, not necessarily convex, $x$ is a fixed point of $K$ if and only if $x \in \mathbb{C}$.

3. If $\mathbb{C}$ is closed and convex, $K(x)$ is closed and convex for any $x \in \operatorname{dom}(K)$.

4. Let $\mathbb{C}$ be closed and convex and $x \in \mathbb{C}$. For this $x$, let $K(x)_{\infty}$ and $\mathbb{C}_{\infty}$ denote the recession cone (see Appendix A.4) of the sets $K(x)$ and $\mathbb{C}$, respectively. Then we have $K(x)_{\infty} \subseteq \mathbb{C}_{\infty}$. Consequently, if $\mathbb{C}$ is bounded, $K(x)$ is bounded for every $x$ in $\mathbb{C}$.

As a consequence of Lemma $5(2)$, the set of fixed points of $K$ is nonempty when $\mathbb{C}$ is nonempty and $\mathrm{QVI}(K, F)$ which seeks such a fixed point as a solution is not vacuous for any such $\mathbb{C}$. Lemma 5(2) can be strengthened significantly: fixed points of $K$ are in the interior of $\mathbb{C}$ if and only if they are in the interior of their image under $K$. This is illustrated below in Fig 1(b) and proved in the following result. See Appendix A.3 for proof. The notation $\operatorname{int}(\bullet)$ and $\partial \bullet$ stand for the interior and the boundary of ' $\bullet$ ' respectively. 
Lemma 6 A point $x$ belongs to the interior of $K(x)$ if and only if $x$ is in the interior of $\mathbb{C}$.

This concludes the preliminaries for this section. The following section addresses the issue of the refinement of the GNE.

\subsection{Refinement of the GNE}

We begin with the formal definition of the refinement.

Definition 2.1 (Refinement) Let $\mathcal{S}$ be a class of generalized Nash games with shared constraints defined by the collection of players, their strategy spaces, their objective functions and the shared constraints. Let $\mathscr{E}(G)$ denote the set of $G N E$ s of a game $G \in \mathcal{S}$ and let $\mathscr{G}$ and $\mathscr{U}$ be defined as

$$
\mathscr{G}:=\{\mathscr{E}(G) \mid G \in \mathcal{S}\} \quad \text { and } \quad \mathscr{U}:=\bigcup_{G \in \mathcal{S}} \mathscr{E}(\mathcal{G})
$$

respectively. A refinement of the GNE of games in $\mathcal{S}$ is a set-valued mapping $\mathcal{R}: \mathscr{G} \rightarrow 2^{\mathscr{U}}$ that satisfies the following properties:

(R1) The refinement of a game must be a subset of equilibria of the game. i.e.

$$
\mathcal{R}(\mathscr{E}(G)) \subseteq \mathscr{E}(G) \quad \forall G \in \mathcal{S}
$$

(R2) Any game with nonempty set of GNEs must admit a refinement. Specifically for all $G \in \mathcal{S}$,

$$
\text { if } \quad \mathscr{E}(G) \neq \emptyset, \quad \text { then } \mathcal{R}(\mathscr{E}(G)) \neq \emptyset .
$$

In studying the $\mathrm{VE}$ as a refinement of the GNE, this paper considers the following rule for generating a refinement of the game $\mathcal{G}$ :

$$
\mathcal{R}(\mathscr{E}(\mathcal{G}))=\mathcal{R}(S O L(Q V I(K, F))):=S O L(V I(\mathbb{C}, F))
$$

Let $\mathcal{S}_{1}$ be the class of generalized Nash games that admit a GNE and let $\mathcal{S}_{2}$ be those that admit a VE. By Theorem 4, we know that $\mathcal{S}_{2} \subseteq \mathcal{S}_{1}$, so $\mathcal{R}$ as defined in (2), satisfies (R1). To confirm the VE as a refinement for games in $\mathcal{S}$ we need that $\mathcal{R}$ satisfies (R2), which, for the game $\mathcal{G}$ from Section 1 amounts to showing

$$
\operatorname{SOL}(\mathrm{QVI}(K, F)) \neq \emptyset \quad \Longrightarrow \quad \operatorname{SOL}(\mathrm{VI}(\mathbb{C}, F)) \neq \emptyset .
$$

If $\widetilde{\mathcal{S}}_{1}$ is the class of games for which a GNE does not exist, the class for which $\mathcal{R}$ is a refinement is given by $\mathcal{S}_{2} \cup \widetilde{\mathcal{S}}_{1}:=\mathcal{S}$.

A natural question one may ask is whether $\mathcal{S}$ is the class of all games. This is answered in the negative by the following counter-example of a game with a (unique) GNE but no VE.

Example 2. Game with unique GNE and no VE: Let $\mathbb{C}=\left\{\left(x_{1}, x_{2}\right) \mid x_{2} \geq e^{-x_{1}}, x_{1} \geq 0\right\}$, and

$$
K(x)=\left\{\left(y_{1}, y_{2}\right) \mid y_{2} \geq e^{-x_{1}}, x_{1} \geq 0, y_{1} \geq 0, x_{2} \geq e^{-y_{1}}\right\}
$$

Let $F(x)=\left(1+x_{1}-\frac{1}{x_{2}}, 1\right)$. It is easily verifed that $x=(0,1)$ satisfies

$$
\left(1+x_{1}-\frac{1}{x_{2}}\right)\left(y_{1}-x_{1}\right)+\left(y_{2}-x_{2}\right) \geq 0 \quad \forall y \in K(x)
$$


and thus $(0,1)$ is a GNE. To show the uniqueness of this GNE, we assume a GNE $x \neq(0,1)$ exists in $\mathbb{C}$ and arrive at a contradiction. For such an $x$, we must have $x_{2} \geq e^{-x_{1}}$, but for this $x$ to be a solution we note that it must satisfy $x_{2}=e^{-x_{1}}$. This follows from the observation that the points $\left\{\left(x_{1}, y_{2}\right) \mid y_{2} \in\left[e^{-x_{1}}, \infty\right)\right\}$ lie in $K(x)$, so if $x_{2}>e^{-x_{1}}$, then the point $y=\left(x_{1}, e^{-x_{1}}\right) \in K(x)$ will not satisfy the QVI condition (4) and thus $x$ cannot solve the QVI. Now since $x_{2}=e^{-x_{1}}$, the point $\left(y_{1}, x_{2}\right)=\left(y_{1}, e^{-x_{1}}\right)$ lies in $K(x)$ for all $y_{1} \in\left[x_{1}, \infty\right)$. If $x$ is a solution of the QVI, for such points we require

$$
\left(1+x_{1}-\frac{1}{x_{2}}\right)\left(y_{1}-x_{1}\right) \geq 0 \quad \forall y_{1} \in\left[x_{1}, \infty\right) .
$$

The term in the first bracket is strictly negative since $x_{2}=e^{-x_{1}}$ and $x \neq(0,1)$, while the term in the second bracket can be made positive for $y>x_{1}$. Thus $x$ cannot be a solution and $(0,1)$ is the only solution. Since every VE is a GNE, this game can have at most one VE, i.e. $(0,1)$. But for $(0,1)$ to be a $\mathrm{VE}$ we require

$$
(0,1)^{T}\left[\left(\begin{array}{l}
y_{1} \\
y_{2}
\end{array}\right)-\left(\begin{array}{l}
0 \\
1
\end{array}\right)\right] \geq 0 \quad \forall y \in \mathbb{C} .
$$

It is easy to check that $y=\left(2, e^{-2}\right) \in \mathbb{C}$ and does not satisfy this. Thus this game has no VE but a unique GNE.

In effect $\mathcal{S}$ is smaller than the class of all generalized Nash games, and therefore our efforts in this paper are focused on identifying subclasses of $\mathcal{S}$. A subclass of $\mathcal{S}_{2}$ is known for which a VE can be shown to exist without using the hypothesis that a GNE exists; we denote this class by $\mathcal{S}_{2}^{\prime}$. Examples of $\mathcal{S}_{2}^{\prime}$ are games where $\mathbb{C}$ is compact or where $F$ is coercive (cf. (11)). For such games we have $\operatorname{SOL}(\operatorname{VI}(\mathbb{C}, F)) \neq \emptyset$ (and hence $\operatorname{SOL}(\mathrm{QVI}(K, F))$ cannot be empty) and (3) holds. Therefore, while identifying subclasses of $\mathcal{S}$, we will also be interested in whether (a) there is any class larger than $\mathcal{S}_{2}^{\prime}$ included in $\mathcal{S}_{2}$ and (b) whether there is any unifying criterion that may be articulated in terms of $F$ and $\mathbb{C}$ that determines $\mathcal{S}$. (a) is answered in the affirmative in Section 2.3, whereas for (b) we see that the Brouwer degree holds promise, in a way made precise below. While the theory we develop focuses on sufficient conditions for a game to have membership in $\mathcal{S}$, for a class of games these conditions are also seen to be necessary. In this development, we ignore settings in which it is possible to claim the existence of a VE independently of the existence of a GNE (i.e. $\mathcal{S}_{2}^{\prime}$ ) and make the VE a refinement by default. Many of these emerge as special cases of our results.

We begin by noting a simple consequence of Lemma 6 - in the interior of $\mathbb{C}$ the GNE and VE are equivalent. Thus, the VE is a refinement for every $\mathcal{G}$ that has a GNE in the interior of $\mathbb{C}$ and this GNE is also a VE. This is established in the theorem below.

Theorem 7 Let $x \in \operatorname{int}(K(x))$. Then $x$ is a $G N E$ of $\mathcal{G}$ if and only if $x$ is a VE.

Proof : Due to Theorem 4, it suffices to prove the "only if" part of the claim. Suppose $x \in \operatorname{int}(K(x))$ is a GNE. By Lemma $6, x \in \operatorname{int}(\mathbb{C})$. It follows that one can construct a ball, $B(x, r)$, centered at $x$ with sufficiently small radius $r$, such that $B(x, r)$ is contained in $K(x) \cap \mathbb{C}$. Since $x$ is a GNE, it follows that

$$
F(x)^{T}(y-x) \geq 0, \quad \forall y \in B(x, r) .
$$

Putting $y=x+r e$ and $y=x-r e$ for an arbitrary unit vector $e$ gives $F(x)^{T} e=0$. Since this holds for each unit vector $e$, we must have $F(x)=0$. As a consequence, $x$ solves $\operatorname{VI}(\mathbb{C}, F)$.

Theorem 7 should not be surprising. If $\mathbb{C}$ is specified using a continuously differentiable algebraic constraint $c(\cdot) \geq 0$, the hypothesis $x \in \operatorname{int}(K(x))$ reduces to $c(x)>0$. If $x$ is a GNE, the Lagrange 
multipliers corresponding to $c(\cdot)>0$ would be zero. $x$ is an equilibrium with shared $(=0)$ multiplier and therefore a VE (see Theorem 15 in Section 3).

Recall the definitions of $\widetilde{\mathbf{F}}_{K}^{\text {nat }}$ and $\mathbf{F}_{\mathbb{C}}^{\text {nat }}$, the natural maps of $\mathrm{QVI}(K, F)$ and $\mathrm{VI}(\mathbb{C}, F)$ from Section 1.1. The result that our theory is built on is Theorem 8. We show that the Brouwer degrees of $\widetilde{\mathbf{F}}_{K}^{\text {nat }}$ and $\mathbf{F}_{\mathbb{C}}^{\text {nat }}$ with respect to zero, whenever well defined, are equal. Recall from Section 1.1 that if $K$ is continuous, $\widetilde{\mathbf{F}}_{K}^{\text {nat }}$ is continuous.

Theorem 8 Let $\Omega$ be an open bounded set such that $\bar{\Omega} \subseteq \operatorname{dom}(K)$ and suppose $K$ is continuous on $\bar{\Omega}$. If $0 \notin \widetilde{\mathbf{F}}_{K}^{\text {nat }}(\partial \Omega)$, then

$$
\operatorname{deg}\left(\widetilde{\mathbf{F}}_{K}^{n a t}, \Omega, 0\right)=\operatorname{deg}\left(\mathbf{F}_{\mathbb{C}}^{n a t}, \Omega, 0\right) .
$$

Proof : First observe that because every VE is a GNE, the assumption that $0 \notin \widetilde{\mathbf{F}}_{K}^{\text {nat }}(\partial \Omega)$ implies that $\mathbf{F}_{\mathbb{C}}^{\text {nat }}$ is not zero on $\partial \Omega$. Thus $\operatorname{deg}\left(\widetilde{\mathbf{F}}_{K}^{\text {nat }}, \Omega, 0\right)$ and $\operatorname{deg}\left(\mathbf{F}_{\mathbb{C}}^{\text {nat }}, \Omega, 0\right)$ are both well defined.

We will use the invariance of the Brouwer degree under homotopy (property 3 of from Section 1.1) to prove the claim. Define $H:[0,1] \times \operatorname{dom}(K): \rightarrow \mathbb{R}^{m}$ as

$$
H(\bar{t}, v)=\bar{t} \mathbf{F}_{\mathbb{C}}^{\text {nat }}(v)+(1-\bar{t}) \widetilde{\mathbf{F}}_{K}^{\text {nat }}(v) \quad \forall \bar{t} \in[0,1], v \in \bar{\Omega} .
$$

By continuity of $K, H$ is a homotopy between $\widetilde{\mathbf{F}}_{K}^{\text {nat }}$ and $\mathbf{F}_{\mathbb{C}}^{\text {nat }}$. By property 3 of the Brouwer degree, if $0 \notin \bigcup_{t \in[0,1]} H(t, \partial \Omega)$, we would have $\operatorname{deg}(H(1, \cdot), \Omega, 0)=\operatorname{deg}(H(0, \cdot), \Omega, 0)$, by which the required result would follow.

We have already seen $0 \notin H(1, \partial \Omega) \cup H(0, \partial \Omega)$. So it suffices that $0 \notin H(\bar{t}, \partial \Omega)$ for all $\bar{t} \in(0,1)$ for the result to follow. Assume that this is not so. i.e. assume that for some $t \in(0,1)$ and $z \in \partial \Omega$, $H(t, z)=0$. Then

$$
z=t x^{c}+(1-t) x^{k},
$$

where $x^{k}=\Pi_{K(z)}(z-F(z))$ and $x^{c}=\Pi_{\mathbb{C}}(z-F(z))$. Since $x^{k} \in K(z),\left(x_{i}^{k}, z^{-i}\right) \in \mathbb{C}$ for every $i \in \mathcal{N}$, implying that the point $x^{a}$ belongs to $\mathbb{C}$, where

$$
x^{a}:=\frac{1}{N} \sum_{i \in \mathcal{N}}\left(x_{i}^{k}, z^{-i}\right)=\frac{(N-1)}{N} z+\frac{1}{N} x^{k} .
$$

Indeed, one may verify that

$$
z=\frac{N(1-t)}{N(1-t)+t} x^{a}+\frac{t}{N(1-t)+t} x^{c},
$$

implying that $z$ is also in $\mathbb{C}$, or equivalently in $K(z)$. Now using the property of projection in Lemma 2 , we get

$$
\left(y-x^{c}\right)^{T}\left(x^{c}-z+F(z)\right) \geq 0 \quad \forall y \in \mathbb{C} \quad \text { and } \quad\left(y-x^{k}\right)^{T}\left(x^{k}-z+F(z)\right) \geq 0, \quad \forall y \in K(z)
$$

Since $z \in K(z) \cap \mathbb{C}$, we may put $y=z$ in both of the above inequalities to get

$$
F(z)^{T}\left(z-x^{c}\right) \geq\left\|z-x^{c}\right\|^{2} \geq 0 \quad \text { and } \quad F(z)^{T}\left(z-x^{k}\right) \geq\left\|z-x^{k}\right\|^{2} \geq 0 .
$$

On the other hand since $z-x^{c}=-\frac{1-t}{t}\left(z-x^{k}\right)$, we have

$$
-\frac{1-t}{t} F(z)^{T}\left(z-x^{k}\right) \geq 0
$$

which from (6) gives $F(z)^{T}\left(z-x^{k}\right)=0$ and $z=x^{k}$. But this means that $\widetilde{\mathbf{F}}_{K}^{\text {nat }}(z)=0$, a contradiction to the hypothesis that $0 \notin \widetilde{\mathbf{F}}_{K}^{\text {nat }}(\partial \Omega)$. Hence $\operatorname{deg}(H(t, \cdot), \Omega, 0)$ is well defined for all $t \in[0,1]$. By property 3 of the Brouwer degree, its value is independent of $t$, whence the result follows. 
The above result is of a deeper flavor than Theorem 4 of Facchinei et al., for it shows a symmetric relationship (equality, rather than a one-way inclusion) between the GNE and the VE. Indeed, it says says that the GNE and the VE are equivalent upto the degree of the corresponding natural maps. Moreover, the only assumptions the theorem makes are those necessary for these degrees to be well defined and the result may thereby be thought of as being germane to such games.

Theorem 8 and the solvability property of the Brouwer degree allow for concluding the validity of the implication in (3) through the degree of $\widetilde{\mathbf{F}}_{K}^{\text {nat }}$. Specifically, if the nonemptiness of $\operatorname{SOL}(\mathrm{QVI}(K, F))$ implies the nonzeroness of $\operatorname{deg}\left(\widetilde{\mathbf{F}}_{K}^{\text {nat }}, \Omega, 0\right)$ for some $\Omega$, i.e. the converse of property 2 of the Brouwer degree holds, then it also implies the nonemptiness of $\mathrm{SOL}(\mathrm{VI}(\mathbb{C}, F))$ and the game admits the VE as a refinement of the GNE. This is articulated in the following theorem.

Theorem 9 Consider game $\mathcal{G}$ and suppose $K$ is continuous. Consider the following statements:

(C0) $\mathcal{G}$ admits a GNE.

(C1) There exists an open bounded set, $\Omega$, with $\bar{\Omega} \subseteq \operatorname{dom}(K)$ such that $\Omega$ contains a GNE of $\mathcal{G}$, and has no $G N E$ of $\mathcal{G}$ on its boundary.

(C2) For an open bounded set, $\Omega$ with $\bar{\Omega} \subseteq \operatorname{dom}(K), \operatorname{deg}\left(\widetilde{\mathbf{F}}_{K}^{n a t}, \Omega, 0\right)$ is well defined and nonzero.

(C3) $\mathcal{G}$ admits a VE.

Then,

(a) we have $\mathbf{( C 2 )} \Longrightarrow(\mathbf{C 3})$. Consequently if $(\mathbf{C 2})$ holds for $\mathcal{G}$, then $\mathcal{G}$ admits a GNE and a VE. $\mathcal{G}$ belongs to the aforementioned class $\mathcal{S}$ and the implication in (3) holds for $\mathcal{G}$.

(b) If $\mathcal{G}$ has the property that

$$
(\mathrm{C} 0) \Longrightarrow(\mathrm{C} 1) \Longrightarrow(\mathrm{C} 2), \text { or } \quad(\mathrm{C} 0) \Longrightarrow(\mathrm{C} 2)
$$

then $\mathcal{G}$ belongs to $\mathcal{S}$ and $(3)$ holds.

\section{Proof :}

(a) Assume (C2) holds. Then by Theorem 8 we get $\operatorname{deg}\left(\mathbf{F}_{\mathbb{C}}^{\text {nat }}, \Omega, 0\right) \neq 0$. By solvability property 2 of the Brouwer degree we conclude that there exists $x \in \Omega$ such that $\mathbf{F}_{\mathbb{C}}^{\text {nat }}(x)=0$. But this means that $x$ solves $\operatorname{VI}(\mathbb{C}, F)$ and is hence a VE. It follows that if (C2) holds for $\mathcal{G}$ then $\mathcal{G}$ admits a GNE and VE and the implication in (3) holds.

(b) If $(\mathbf{C 0}) \Longrightarrow(\mathbf{C 1}) \Longrightarrow(\mathbf{C 2})$ or if $(\mathbf{C 0}) \Longrightarrow(\mathbf{C 2})$, then we may say that $(\mathbf{C 0})$, i.e. existence of a solution to $\operatorname{QVI}(K, F)$, is sufficient for $\operatorname{deg}\left(\widetilde{\mathbf{F}}_{K}^{\text {nat }}, \Omega, 0\right)$ to be nonzero. Then using part (a), we conclude (C0) implies that $\mathcal{G}$ admits a VE. Thus, for $\mathcal{G}$, the existence of a GNE is a sufficient condition for the existence of a VE and (3) holds.

The next theorem shows that for a class of games, (a) and (b) are not just sufficient but also necessary for (3) to hold. This indicates that using Theorem 9 to claim (3) is an apt approach.

Theorem 10 Let $K$ be continuous, $S O L(Q V I(K, F))$ be bounded and $\Omega$ be an open bounded set with $\bar{\Omega} \subseteq \operatorname{dom}(K)$ containing $\operatorname{SOL}(Q V I(K, F))$. If $F$ is pseudo-monotone ${ }^{1}$, then the implication

$$
\operatorname{SOL}(\mathrm{QVI}(K, F)) \neq \emptyset \Longrightarrow \operatorname{SOL}(\mathrm{VI}(\mathbb{C}, F)) \neq \emptyset
$$

holds if and only if the following implication holds.

$$
\operatorname{SOL}(\operatorname{QVI}(K, F)) \neq \emptyset \Longrightarrow \operatorname{deg}\left(\widetilde{\mathbf{F}}_{K}^{n a t}, \Omega, 0\right) \neq 0 .
$$

\footnotetext{
${ }^{1} \mathrm{~A}$ mapping $F: \mathbb{R}^{m} \rightarrow \mathbb{R}^{m}$ is said to be pseudo-monotone if for all $x, y \in \mathbb{R}^{m}, F(y)^{T}(x-y) \geq 0 \Longrightarrow F(x)^{T}(x-y) \geq 0$.
} 
Proof : We first recall Theorem 2.3.17 in [7] that since the solution set of $\mathrm{VI}(\mathbb{C}, F)$ (if nonempty) is bounded and $F$ is pseudo-monotone,

$$
\operatorname{SOL}(\operatorname{VI}(\mathbb{C}, F)) \neq \emptyset \Longleftrightarrow \operatorname{deg}\left(\mathbf{F}_{\mathbb{C}}^{\text {nat }}, \Omega, 0\right) \neq 0 .
$$

The proof of the result is now easy to see. Suppose (7) holds. Then combining (7), (9) and Theorem 8 , we get (8). Conversely, if (8) holds, then using Theorem 8 we get

$$
\operatorname{SOL}(\operatorname{QVI}(K, F)) \cap \Omega \neq \emptyset \Longrightarrow \operatorname{deg}\left(\mathbf{F}_{\mathbb{C}}^{\text {nat }}, \Omega, 0\right) \neq 0,
$$

which by the solvability property of the Brouwer degree leads to (7).

Theorem 9 forms the basis for identifying subclasses of $\mathcal{S}$. It provides a framework, via (C1) and (C2), for identifying games for which the implication $(\mathbf{C 0}) \Longrightarrow$ (C3) holds. More complex chains of implications than those in (a) and (b) may also be framed using (C0) - (C3) for this purpose. Note that (C2) does not follow from (C1), since in general the converse of solvability property 2 of the Brouwer degree does not hold.

We now apply Theorem 9 to identify subclasses of $\mathcal{S}$. A sufficient condition for $\mathcal{G}$ to satisfy, "(C0) $\Longrightarrow(\mathbf{C 1}) \Longrightarrow(\mathbf{C 2})$ " is that $\widetilde{\mathbf{F}}_{K}^{\text {nat }}$ be continuous and one-to-one. But since generalized Nash games are known to have manifolds of equilibria, we expect $\widetilde{\mathbf{F}}_{K}^{\text {nat }}$ to have manifolds of zeros in fairly general cases (see Theorem 20). Consequently, it is unlikely that $\widetilde{\mathbf{F}}_{K}^{\text {nat }}$ can be shown to be one-to-one in general and we do not attempt that line of research. Instead we observe that in (C2) (and in (8)), we may ask that $\operatorname{deg}\left(\mathbf{F}_{\mathbb{C}}^{\text {nat }}, \Omega, 0\right)$ be nonzero, thanks to Theorem 4 , and use this to identify these subclasses. We begin by showing that certain classes in $\mathcal{S}_{2}^{\prime}$ are identified by Theorem 9 (where $\mathcal{S}_{2}^{\prime}$ denotes the subset of $\mathcal{S}_{2}$ for which the existence of the VE can be shown without the knowledge of the existence of a GNE). In particular, we see that Theorem 9 includes in it the well known fact: if $\mathbb{C}$ is compact, the VE and GNE both exist and hence the VE is a refinement. Next we show Theorem 9 contains games where $F$ is a coercive mapping as a special case. In both of these proofs we will consider an open bounded set $\Omega \subseteq \mathbb{R}^{m}$ and consider the homotopy $H:[0,1] \times \bar{\Omega} \rightarrow \mathbb{R}^{m}$ given by

$$
H(t, x)=x-\Pi_{\mathbb{C}}\left(t(x-F(x))+(1-t) x^{\mathrm{ref}}\right) \quad \forall x \in \bar{\Omega}, t \in[0,1],
$$

for a specific choice of $x^{\text {ref }} \in \Omega \cap \mathbb{C}$. The hypotheses of the particular result are then shown to imply that $H(1, \cdot)=\mathbf{F}_{\mathbb{C}}^{\text {nat }}$ is homotopic to $H(0, \cdot)=\mathbf{1}-x^{\text {ref }}$, which by property 3 of the Brouwer degree gives $\operatorname{deg}\left(\mathbf{F}_{\mathbb{C}}^{\text {nat }}, \Omega, 0\right)=1$. Note that the existence of the GNE, i.e.(C0), is not used and (C2) is claimed directly.

Lemma 11 (Brouwer's fixed point theorem) Let $\mathbb{C}$ be compact and suppose $\Omega$ is an open bounded set large enough to strictly contain $\mathbb{C}$. Then $\operatorname{deg}\left(\mathbf{F}_{\mathbb{C}}^{\text {nat }}, \Omega, 0\right)=1$ and the game admits a VE and a GNE.

Proof : Let $x^{\text {ref }} \in \mathbb{C}$ be some point and for $\Omega$ as given, consider the homotopy $H$ in (10). Since $\Omega$ strictly contains $\mathbb{C}, \partial \Omega \cap \mathbb{C}=\emptyset$. Since for any $t$, zeros of $H(t, \cdot)$ lie in $\mathbb{C}$, we must have $H(t, \partial \Omega) \neq 0$ for every $t \in[0,1]$. By property 3 of the Brouwer degree, $\operatorname{deg}\left(\mathbf{F}_{\mathbb{C}}^{\text {nat }}, \Omega, 0\right)=\operatorname{deg}\left(\mathbf{1}-x^{\mathrm{ref}}, \Omega, 0\right)=1$, where the last equality is because $x^{\text {ref }}$ belongs to $\mathbb{C} \subset \Omega$. By the solvability property 2 of the Brouwer degree, the game admits a VE. Now we may use Theorem 4 to conclude that the game also admits a GNE.

We next see that if $F$ is coercive, then the VE is a refinement. The proof below is adapted from [7, Proposition 2.2.3].

Lemma 12 Suppose there exists an $x^{r e f} \in \mathbb{C}$ such that

$$
\liminf _{x \in \mathbb{C},\|x\| \rightarrow \infty} F(x)^{T}\left(x-x^{r e f}\right)>0 .
$$

There exists an open bounded set $\Omega$ such that $\operatorname{deg}\left(\mathbf{F}_{\mathbb{C}}^{\text {nat }}, \Omega, 0\right)=1$ and this game has a VE and GNE. 
Proof : Since the limit above is positive, there exists an open bounded set $\Omega$ such that $F(x)^{T}\left(x-x^{\text {ref }}\right)>$ 0 for all $x \in \partial \Omega \cap \mathbb{C}$. Without loss of generality one may take $\Omega$ large enough to contain $x^{\text {ref }}$. Since $x^{\text {ref }} \in \mathbb{C}$, it follows that no point on the set $\partial \Omega \cap \mathbb{C}$ solves $\operatorname{VI}(\mathbb{C}, F)$. Let $H$ be as in $(10)$. It is easy to see that $0 \notin H(1, \partial \Omega) \cup H(0, \partial \Omega)$. Assume that $H(t, x)=0$ for some $t \in(0,1)$ and $x \in \partial \Omega$. Since zeros of $H(t, \cdot)$ must lie in $\mathbb{C}, x \in \partial \Omega \cap \mathbb{C}$. Now by Lemma 2

$$
(y-x)^{T}\left(x-t(x-F(x))-(1-t) x^{\mathrm{ref}}\right) \geq 0 \quad \forall y \in \mathbb{C},
$$

whereby for $y=x^{\text {ref }}$,

$$
F(x)^{T}\left(x-x^{\mathrm{ref}}\right)<-\frac{1-t}{t}\left\|x-x^{\mathrm{ref}}\right\|^{2}<0 .
$$

Since $x \in \partial \Omega$, this is a contradiction. Consequently, our assumption that $H(t, x)=0$ is incorrect and we must have $0 \notin \bigcup_{t \in[0,1]} H(t, \partial \Omega)$. Therefore property 3 of the Brouwer degree $\operatorname{deg}\left(\mathbf{F}_{\mathbb{C}}^{\text {nat }}, \Omega, 0\right)=$ $\operatorname{deg}\left(\mathbf{1}-x^{\mathrm{ref}}, \Omega, 0\right)=1$, whereby this game has a VE and by Theorem 4, a GNE.

Notice that since in Lemma 11 and Lemma 12 we are essentially proving $\operatorname{deg}\left(\mathbf{F}_{\mathbb{C}}^{\text {nat }}, \Omega, 0\right)$ to be nonzero, the requirement of the continuity of $K$ imposed in Theorem 8 has been relaxed and we have instead argued the existence of the GNE using Theorem 4 after proving the existence of a VE as above.

We now turn to an existence result for QVIs that claims (C2), and thus provides a sufficient condition for the VE to be a refinement of the GNE. This result is Corollary 2.8.4 in [7, page 222], which applies to any QVI, not necessarily those arising from $K$ as defined as in (1). We have reproduced it below with the added hypothesis " $0 \notin \widetilde{\mathbf{F}}_{K}^{\text {nat }}(\partial \Omega)$ ".

Theorem 13 (Theorem 2.8.3 and Corollary 2.8.4 [7]) Let $K: \mathbb{R}^{m} \rightarrow 2^{\mathbb{R}^{m}}$ be a closed-valued and convex-valued point-to-set map. Let $F: \mathbb{R}^{m} \rightarrow \mathbb{R}^{m}$ be a continuous function. Suppose there exists an open bounded set $\Omega \subset \operatorname{dom}(K)$ and a vector $x^{\text {ref }} \in \Omega$ such that $0 \notin \widetilde{\mathbf{F}}_{K}^{\text {nat }}(\partial \Omega)$ and

1. $K$ is continuous on $\bar{\Omega}$

2. $x^{\text {ref }}$ belongs to $K(x)$ for every $x \in \bar{\Omega}$

3. the following holds

$$
\left\{x \in K(x):\left(x-x^{r e f}\right)^{T} F(x)<0\right\} \cap \partial \Omega=\emptyset .
$$

Then $\operatorname{deg}\left(\widetilde{\mathbf{F}}_{K}^{\text {nat }}, \Omega, 0\right) \neq 0$.

For a shared constraint game such as $\mathcal{G}$, condition (3) in Theorem 13 above is equivalent to requiring that

$$
\left\{x \in \mathbb{C}:\left(x-x^{\mathrm{ref}}\right)^{T} F(x)<0\right\} \cap \partial \Omega=\emptyset,
$$

since by using Lemma $5(2), x \in K(x) \Longleftrightarrow x \in \mathbb{C}$. This in turn is known to be a sufficient condition for $\operatorname{deg}\left(\mathbf{F}_{\mathbb{C}}^{\text {nat }}, \Omega, 0\right)$ to be well defined and nonzero, by Lemma 12 . In this way, Theorem 8 leads to an alternative proof for the above theorem for $K$ as in (1) and unifies Proposition 2.2.3 and Corollary 2.8.4 in [7] which are the chief existence results for VIs and QVIs respectively.

\section{$2.3 \quad$ Identification of subclasses of $\mathcal{S} \backslash \mathcal{S}_{2}^{\prime}$}

In section we concentrate on the identification of classes of games in $\mathcal{S}_{2}$ that do not lie in $\mathcal{S}_{2}^{\prime}$. We show by an argument based on Theorem 9 , that the class of games with pseudo-monotone $F$ and with certain other properties of the recession cone of $\mathbb{C}$ have the $\mathrm{VE}$ as a refinement. Recall that in the proof of Theorem 10 we had seen that if $F$ is pseudo-monotone and $\operatorname{SOL}(\operatorname{VI}(\mathbb{C}, F))$ is nonempty and bounded then $\operatorname{deg}\left(\mathbf{F}_{\mathbb{C}}^{\text {nat }}, \Omega, 0\right)$ is well defined and nonzero over any neighbourhood $\Omega$ containing $\operatorname{SOL}(\operatorname{VI}(\mathbb{C}, F))$. 
Our next result is an "extension" of this fact to QVI $(K, F)$ : for a certain class of games (C0) implies that either (C3) holds or (C2) holds, and as a consequence $(\mathbf{C 0}) \Longrightarrow$ (C3). Definitions of the normal cone $(\mathscr{N})$, tangent cone $(\mathscr{T})$, recession cone $\left(\mathbb{C}_{\infty}\right)$ and dual cone (denoted by $S^{*}$ for a cone $S$ ) used below can be found in Appendix A.4.

Theorem 14 Suppose $F$ is pseudo-monotone and $x^{\text {ref }}$ is a GNE of $\mathcal{G}$. Consider the following conditions:

(1) Either $F\left(x^{r e f}\right)=0$ or $-F\left(x^{r e f}\right) \in \operatorname{int}\left(\mathscr{N}\left(x^{r e f} ; K\left(x^{r e f}\right)\right)\right)$,

(1') $\mathscr{T}\left(x^{r e f} ; K\left(x^{r e f}\right)\right)^{*} \backslash\{0\} \subseteq \operatorname{int}\left(\mathbb{C}_{\infty}^{*}\right)$,

(2) $\mathbb{C}_{\infty} \subseteq \mathscr{T}\left(x^{r e f} ; K\left(x^{r e f}\right)\right)$.

If $\mathcal{G}$ has the property that condition (2) holds and either (1) or (1') holds then $\mathcal{G}$ admits a VE.

Proof : Our result is proved through the following set of steps.

Step 1: If $\mathbb{C}$ is bounded there is nothing to prove, cf. Lemma 11 , so we assume that $\mathbb{C}$ is unbounded

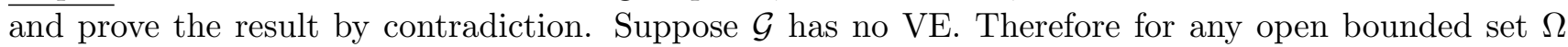
containing $x^{\text {ref }}, 0 \notin \mathbf{F}_{\mathbb{C}}^{\text {nat }}(\partial \Omega)$. Fix such an $\Omega$ and consider the homotopy $H$ from (10). Since $x^{\text {ref }} \in \Omega$, we have $0 \notin H(0, \partial \Omega) \cup H(1, \partial \Omega)$. If we have $0 \notin \bigcup_{t \in(0,1)} H(t, \partial \Omega)$, then we would get $\operatorname{deg}\left(\mathbf{F}_{\mathbb{C}}^{\text {nat }}, \Omega, 0\right)=$ $\operatorname{deg}\left(\mathbf{1}-x^{\text {ref }}, \Omega, 0\right)=1$, implying that $\mathcal{G}$ has a VE in $\Omega$. This contradicts our assumption. So we must have $H(t, x)=0$ for some $x \in \partial \Omega \cap \mathbb{C}$ and $t \in(0,1)$. i.e. for such $x, t$ we have $x=\Pi_{\mathbb{C}}\left(t(x-F(x))+(1-t) x^{\mathrm{ref}}\right)$. Therefore by Lemma 2 ,

$$
(y-x)^{T}\left(x-t(x-F(x))-(1-t) x^{\mathrm{ref}}\right) \geq 0 \quad \forall y \in \mathbb{C} .
$$

As in Lemma 12 , putting $y=x^{\text {ref }}$ gives

$$
F(x)^{T}\left(x-x^{\mathrm{ref}}\right)<-\frac{1-t}{t}\left\|x-x^{\mathrm{ref}}\right\|^{2}<0 .
$$

Now since $F$ is pseudo-monotone, it follows that $F\left(x^{\mathrm{ref}}\right)^{T}\left(x-x^{\mathrm{ref}}\right) \leq 0$. Since $\Omega$ was arbitrary, we conclude that for each open bounded set $\Omega$ containing $x^{\text {ref }}$, there exists an $x \in \partial \Omega \cap \mathbb{C}$ such that $F\left(x^{\mathrm{ref}}\right)^{T}\left(x-x^{\mathrm{ref}}\right) \leq 0$.

Step 3: Let $\left\{\Omega_{k}\right\}$ be a sequence of increasing open balls, each containing $x^{\text {ref }}$, such that $\bigcup_{k \in \mathbf{N}} \Omega_{k}=\mathbb{R}^{m}$. $\overline{\text { Let } x_{k}} \in \partial \Omega_{k} \cap \mathbb{C}$ be such that $F\left(x^{\mathrm{ref}}\right)^{T}\left(x_{k}-x^{\mathrm{ref}}\right) \leq 0$. Assume, without loss of generality, that the sequence $\left\{\frac{x_{k}-x^{\mathrm{ref}}}{\left\|x_{k}-x^{\mathrm{ref}}\right\|}\right\}$ is convergent and let its limit be $d^{\prime}$. We have $d^{\prime} \neq 0$ and

$$
F\left(x^{\mathrm{ref}}\right)^{T} d^{\prime} \leq 0
$$

Step 4: Next, we prove that $d^{\prime} \in \mathbb{C}_{\infty}$. Let $\tau \geq 0$ be arbitrary. Since $\left\|x_{k}\right\| \rightarrow \infty$, for sufficiently large $k$,

$$
\frac{\tau}{\| x_{k}-x^{\mathrm{ref} \|}} \in[0,1], \quad \text { whereby } \quad u_{k}:=x^{\mathrm{ref}}+\tau \frac{x_{k}-x^{\mathrm{ref}}}{\left\|x_{k}-x^{\mathrm{ref}}\right\|} \in \mathbb{C} .
$$

By closedness of $\mathbb{C}, \lim _{k \rightarrow \infty} u_{k}=x^{\mathrm{ref}}+\tau d^{\prime} \in \mathbb{C}$. Since $\tau$ is arbitrary and $\mathbb{C}$ is convex, $d^{\prime}$ is a recession direction of $\mathbb{C}$.

Step 5: To finish the proof, recall that the normal cone and the tangent cone of a convex set are related in the following way $[23,7]$

$$
-\mathscr{N}\left(x^{\mathrm{ref}} ; K\left(x^{\mathrm{ref}}\right)\right)=\mathscr{T}\left(x^{\mathrm{ref}} ; K\left(x^{\mathrm{ref}}\right)\right)^{*} \text {. }
$$


Furthermore, since $x^{\text {ref }}$ is a GNE of $\mathcal{G}, F\left(x^{\text {ref }}\right) \in \mathscr{T}\left(x^{\text {ref }} ; K\left(x^{\text {ref }}\right)\right)^{*}$. Suppose (1) holds. If $F\left(x^{\text {ref }}\right)=0$ then $x^{\text {ref }}$ itself is a VE and there is nothing to prove. If $-F\left(x^{\text {ref }}\right) \in \operatorname{int}\left(\mathscr{N}\left(x^{\text {ref }} ; K\left(x^{\text {ref }}\right)\right)\right)$ then for all nonzero vectors $d$ in $\mathscr{T}\left(x^{\text {ref }} ; K\left(x^{\text {ref }}\right)\right)$ we must have

$$
F\left(x^{\mathrm{ref}}\right)^{T} d>0 .
$$

But because (2) holds, (13) must also hold for all nonzero $d$ in $\mathbb{C}_{\infty}$. Putting $d=d^{\prime}$ in (13) contradicts (12). Now suppose condition $\left(1^{\prime}\right)$ holds. Then $F\left(x^{\text {ref }}\right) \in \operatorname{int}\left(\mathbb{C}_{\infty}^{*}\right)$ and so $(13)$ is satisfied by $d=d^{\prime}$; a contradiction to (12) is reached. Thus our initial assumption is incorrect; $\mathcal{G}$ must admit a VE.

Note that the pseudo-monotonicity of $F$ and the properties of $\mathbb{C}$ mentioned in Theorem $14\left(1^{\prime}\right),(2)$ are by themselves insufficient for the existence of a VE of $\mathcal{G}$. But given that a GNE $x^{\text {ref }}$ exists, the above theorem provides sufficient conditions for $\mathcal{G}$ to have a VE. The above theorem is thus seen to identify a class of games lying in $\mathcal{S} \backslash \mathcal{S}_{2}^{\prime}$. It is not hard to see that (2) is satisfied in two cases in which we have already seen the VE to be a refinement: the case where $\mathbb{C}$ is compact (in this case $\mathbb{C}_{\infty}=\{0\}$ and is included in any cone; herein (1') from Theorem 14 also holds) and the case where $x^{\mathrm{ref}} \in \operatorname{int}\left(K\left(x^{\mathrm{ref}}\right)\right)$ (here $\mathscr{T}\left(x^{\text {ref }} ; K\left(x^{\text {ref }}\right)\right)=\mathbb{R}^{m}$ and (1) from Theorem 14 holds, since $\left.F\left(x^{\text {ref }}\right)=0\right)$. Furthermore condition (1) is necessary, since the existence of a VE necessitates the existence of a GNE satisfying (1) (take $x^{\text {ref }}$ $=\mathrm{VE}$ ). Next, we discuss some instances where the above sufficiency condition may be applied.

Example 3. Generalized Nash game with affine shared constraints: Consider a game $\mathcal{G}$ where $F$ is pseudo-monotone and $\mathbb{C}=\{x \mid A x \geq b, x \geq 0\}$ for some nonnegative $b \in \mathbb{R}^{n}$ and $n \times m$ matrix $A$ with nonnegative elements. Let $x^{\text {ref }}$ be a GNE such that $A x^{\text {ref }}=b$. Suppose $A=\left[a_{1}, \ldots, a_{N}\right]$, where $a_{i} \in \mathbb{R}^{n \times m_{i}}$. We have $\mathbb{C}_{\infty}=\{d \mid A d \geq 0, d \geq 0\}$ implying that $\mathbb{C}_{\infty}^{*}=\left\{A^{T} \lambda \mid \lambda \geq 0\right\}$. Then $K\left(x^{\text {ref }}\right)$ and $\mathscr{T}\left(x^{\text {ref }} ; K\left(x^{\text {ref }}\right)\right)$ are given as

$$
K\left(x^{\mathrm{ref}}\right)=\prod_{i \in \mathcal{N}}\left\{y_{i} \mid a_{i} y_{i}+\sum_{j \neq i} a_{j} x_{j}^{\mathrm{ref}} \geq b, y_{i} \geq 0\right\}, \quad \mathscr{T}\left(x^{\mathrm{ref}} ; K\left(x^{\mathrm{ref}}\right)\right)=\prod_{i \in \mathcal{N}}\left\{d_{i} \mid a_{i} d_{i} \geq 0, d_{i} \geq 0\right\} .
$$

Clearly, $\mathscr{T}\left(x^{\text {ref }} ; K\left(x^{\text {ref }}\right)\right) \subseteq \mathbb{C}_{\infty}$. But by noting that $A$ has nonnegative entries, we have that $\mathbb{C}_{\infty}=$ $\mathscr{T}\left(x^{\text {ref }} ; K\left(x^{\text {ref }}\right)\right)$. Therefore if (1) from Theorem 14 holds, we conclude from Theorem 14 that the game $\mathcal{G}$ also admits a VE.

Example 4. Generalized Nash game with non-affine shared constraints: Evidently, Theorem 14 can apply to numerous other games with non-affine constraints since requirement (1') from Theorem 14 is not very restrictive but the lack of a general expression for $\mathbb{C}_{\infty}$ makes it harder to provide examples. To illustrate games where (1') from Theorem 14 may hold, we present the following example in $\mathbb{R}^{2}$. Let $F: \mathbb{R}^{2} \rightarrow \mathbb{R}^{2}$ be pseudo-monotone, $\mathbb{C}=\left\{\left(x_{1}, x_{2}\right) \mid x_{1}^{2}-2 x_{1} x_{2}+x_{2}^{2}-x_{1} \sqrt{2}-x_{2} \sqrt{2} \leq 0\right\}$, be the epigraph of a tilted parabola. Thus $\mathbb{C}_{\infty}=\left\{\left(x_{1}, x_{2}\right) \mid x_{1}=x_{2} \geq 0\right\}$, whereas for $x^{\text {ref }}$ as shown in Fig 1 , $\mathscr{T}\left(x^{\mathrm{ref}} ; K\left(x^{\mathrm{ref}}\right)\right)=[0, \infty) \times[0, \infty)$. Thus Theorem $14\left(1^{\prime}\right)$ holds (so Theorem $14(2)$ also holds). Hence if $x^{\text {ref }}$ is a GNE, $\mathcal{G}$ admits a VE.

Following are some concluding remarks about the above result. Theorem 14 can be claimed via Theorem 2.3.5, in [7, page 158]. We have avoided that path in order to demonstrate the reach of Theorem 9. The 'int' in conditions (1) and (1') presupposes that cones $\mathscr{N}\left(x^{\mathrm{ref}} ; K\left(x^{\mathrm{ref}}\right)\right)$ and $\mathbb{C}_{\infty}^{*}$ have an interior. The 'int' may be relaxed to 'relative interior' if the cones satisfy some regularity; see [7, ch. 2.4.1] for details. It is easy to show (see, e.g., [24]) that

$$
\mathscr{N}\left(x^{\mathrm{ref}} ; K\left(x^{\mathrm{ref}}\right)\right)=\prod_{i \in \mathcal{N}} \mathscr{N}\left(x_{i}^{\mathrm{ref}} ; K_{i}\left(x^{\mathrm{ref},-i}\right)\right) .
$$




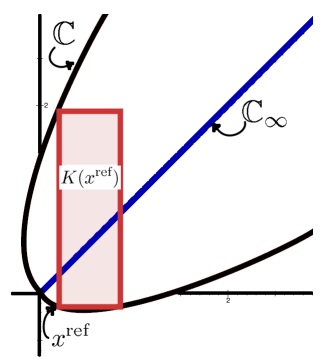

Figure 1: Example where Theorem 14(1') holds

When $\mathbb{C}$ is given as an algebraic constraint $\{v \mid c(v) \geq 0\}$ for a continuously differentiable function $c$ : $\mathbb{R}^{m} \rightarrow \mathbb{R}^{n}$ and $x^{\text {ref }}$ is on the boundary of $\mathbb{C}, \mathscr{N}\left(x_{i}^{\text {ref }} ; K_{i}\left(x^{\text {ref, }-i}\right)\right)=\left\{\alpha^{T} \nabla_{i} c\left(x^{\text {ref }}\right) \mid \alpha \in \mathbb{R}^{n} \geq 0\right\}$. Hence $\mathscr{N}\left(x^{\mathrm{ref}} ; K\left(x^{\mathrm{ref}}\right)\right)$ is at most $n N$ dimensional. In this setting, for $\mathscr{N}\left(x^{\mathrm{ref}} ; K\left(x^{\mathrm{ref}}\right)\right)$ to have a nonempty interior, it is necessary that $m \leq n N$. When we do have $\operatorname{int}\left(\mathscr{N}\left(x^{\text {ref }} ; K\left(x^{\text {ref }}\right)\right)\right) \neq \emptyset$, Theorem $14(1)$ has another interpretation. It says that either $F\left(x^{\mathrm{ref}}\right)=0$ or the Lagrange multipliers corresponding to the constraint $c$ are all strictly positive at $x^{\text {ref }}$. Interestingly, condition (1) in Theorem 14 and the requirement that $m \leq n N$ also appear in the sufficient condition for the existence of a manifold of GNEs (Theorem 20 in Section A.1). The connections between Theorems 14 and 20 are being studied further as part of ongoing research.

Review of sufficiency conditions: We now summarize the contributions of this section. The natural maps corresponding to the GNE and the VE were shown to have equal Brouwer degree (cf. Theorem 8). Therefore a sufficient condition for a class (denoted $\mathcal{S}$ ) to have the VE as a refinement of the GNE is that the existence of a GNE imply the nonzeroness of the degree of these natural maps (cf. Theorem 9). This condition is also necessary for a certain class of games (cf. Theorem 10). We divide $\mathcal{S}$ into further subclasses: a class (denoted by $\mathcal{S}_{2}^{\prime}$ ) with properties such that the existence of the VE can be claimed independently of the knowledge of the GNE; and the class where the VE exists if a GNE exists $\left(\mathcal{S} \backslash \mathcal{S}_{2}^{\prime}\right)$. Lemmas 11 and 12 showed that classes in $\mathcal{S}_{2}^{\prime}$, i.e. the class of games with compact $\mathbb{C}$ and those with a coercive $F$, are special cases of Theorem 9 and thus admit the VE as a refinement of the GNE. Subclasses of $\mathcal{S} \backslash \mathcal{S}_{2}^{\prime}$ were identified in Theorem 14 as games with a pseudo-monotone mapping $F$ and with recession cones $\mathbb{C}_{\infty}$ admitting certain properties. In summary, we have developed a host of broad verifiable conditions for claiming whether shared-constraint Nash games can admit a VE as a refinement of the GNE.

\section{Primal-dual Generalized Nash and Variational Equilibria}

We now pursue the degree theoretic approach to the question of the refinement of the GNE in the primal-dual space. Throughout, we assume an algebraic form for the constraint set $\mathbb{C}$ and assume that an appropriate constraint qualification holds. The nonlinear equations whose zero is the GNE (in the primal-dual space), are, expectedly, given by the natural map of a complementarity problem (CP). In our formulation, the nonlinear equations for the $\mathrm{VE}$ are the natural map of a $\mathrm{CP}$ with additional linear constraints (the reason for this will clarified below). Using these maps, we show that the degree theoretic approach of the previous section has a natural extension to the primal-dual space. While this extension may be intuitively expected, it must be emphasized that it is not a corollary of the primal approach. The maps used in the primal setting $\left(\mathbf{F}_{\mathbb{C}}^{\text {nat }}\right.$ and $\left.\widetilde{\mathbf{F}}_{K}^{\text {nat }}\right)$ and the ones we use in the primal-dual setting ( $\mathbf{G}^{\text {nat }}$ and $\mathbf{J}^{\text {nat }}$ ) are very different (and defined on different spaces) and there is no obvious degree theoretic connection that one can draw between them. 
The primal-dual characterization offers various advantages over the primal characterization. For instance, the assumptions are less abstract and easier to verify; the constraints are assumed to have continuously differentiable boundaries of algebraic form, as opposed to the assumption of continuity of the set-valued map $K$. The analysis is simplified since we escape the QVI setting and now work with pure CPs or mixed CPs. As a result the well-formed theory of VIs and CPs can be used to our advantage. Moreover the set over which these CPs defined are cartesian products of sets (in the primal-dual space), i.e. these CPs are are cartesian VIs or partitioned VIs for which numerous results are known that are simpler than those for general VIs.

We assume, unless mentioned otherwise, that $\mathbb{C}=\{x \mid x \geq 0, c(x) \geq 0\}$ where $c: \mathbb{R}^{m} \rightarrow \mathbb{R}$ is a concave continuously differentiable function. $c$ is assumed $\mathbb{R}$-valued as opposed to $\mathbb{R}^{n}$-valued, only to ease the exposition; in most cases, no generality is lost. We will make the generality or the absence thereof clear wherever necessary. Recall optimization problems $\mathrm{A}_{i}$ from Section 1. Suppose an appropriate constraint qualification holds (see [7]). Then the Karush-Kuhn-Tucker (KKT) conditions for optimality of $x_{i}$ for $\mathrm{A}_{i}\left(x^{-i}\right)$ are given by

$$
\begin{aligned}
& 0 \leq x_{i} \perp \nabla_{i} \varphi_{i}(x)-\lambda_{i} \nabla_{i} c(x) \geq 0 \\
& 0 \leq \lambda_{i} \perp c(x) \geq 0,
\end{aligned}
$$

where $\lambda_{i}$ are the Lagrange multipliers for $\mathrm{A}_{i}$ corresponding to the constraint $c(\cdot) \geq 0$. For $u, v \in \mathbb{R}^{n}$, the notation $0 \leq u \perp v \geq 0$ means $u, v \geq 0$ and $u_{i} v_{i}=0$ for $i=1, \ldots, n$. A strategy tuple $x$ is a GNE of $\mathcal{G}$ if there exist $\lambda_{1}, \ldots, \lambda_{N}$ that together with $x$ simultaneously satisfy the $N$ systems $\left\{\mathrm{KKT}_{i}\right\}_{i \in \mathcal{N}}$. The VE can also be interpreted in terms of the KKT systems, as observed by Facchinei et al. [5].

Theorem 15 (Theorem 3.1 [5]) Let $x$ be a GNE for which the system $\left\{K K T_{i}\right\}_{i \in \mathcal{N}}$ is satisfied with $\lambda_{1}=\lambda_{2}=\cdots=\lambda_{N}$. Then $x$ solves $\operatorname{VI}(\mathbb{C}, F)$. Conversely if $x$ solves $\operatorname{VI}(\mathbb{C}, F)$ then there exist $\lambda \in \mathbb{R}$ such that $\left\{K K T_{i}\right\}_{i \in \mathcal{N}}$ hold with $\lambda_{i}=\lambda$ for all $i \in \mathcal{N}$.

Proof : The proof follows exactly the same argument as in Theorem 3.1 [5].

This theorem sheds new light on equilibria with "shared multipliers" and "non-shared multipliers" for games with shared constraints. Historically, if there existed $\left(x, \lambda_{1}, \ldots, \lambda_{N}\right) \in \mathbb{R}^{m+N}$ that satisfied $\left\{\mathrm{KKT}_{i}\right\}_{i \in \mathcal{N}}, x$ was is called a GNE of $\mathcal{G}$ with non-shared multipliers. If for some $x \in \mathbb{R}^{m}$ and $\lambda \in \mathbb{R}$, $\left\{\mathrm{KKT}_{i}\right\}_{i \in \mathcal{N}}$ were met with $\lambda_{1}=\lambda_{2}=\ldots=\lambda_{N}=\lambda$, then $x$ was called a GNE of $\mathcal{G}$ with shared multipliers. Rosen [25] was the first to spot the possibility of redundancy of multipliers when the constraint was shared - he termed the shared multiplier GNE as a normalized equilibrium. The fact that the GNE with shared multipliers was actually a solution of a related VI was a new insight in the context of such games.

With this understanding, the idea of the VE as a refinement of GNE can now be cast differently. The class of games $\mathcal{S}$ for which the implication in (3) holds are those for which the existence of GNE with non-shared multipliers implies the existence of a GNE with shared multipliers. This property also has an interesting economic interpretation. In many games with shared constraints, such as a bandwith sharing game [14], the Lagrange multipliers corresponding to the constraint can be interpreted as the "price" charged on a player for using a particular strategy by an administrator who controls the shared constraint. Thus the equilibrium with non-shared multipliers can be interpreted as an equilibrium resulting from discriminatory prices, i.e. prices that are charged by an administrator who may discriminate between various users of his constraint. But often the situation modeled makes it unrealistic for the administrator to be able to distinguish between users and the economically appropriate equilibrium is one in which the same price is charged to all users, i.e. a shared multiplier equilibrium. The question of the refinement assumes immediate relevance here. When does an administrator always 
have the option of charging a uniform price across all users to enforce equilibrium? If the implication in (3) holds, we can say that whenever an equilibrium with discriminatory prices exists, one with uniform prices also exists. Moreover, if an equilibrium with uniform prices does not exist, no equilibrium exists.

\subsection{Refinement of the primal-dual GNE}

A result similar to Theorem 8 is obtainable by invoking KKT conditions for characterizing GNE and VE. Recall the systems $(\mathrm{KKT})_{i}$, denote by $\Lambda$ the tuple $\left(\lambda_{1}, \ldots, \lambda_{N}\right)$ and define $\mathbf{G}^{\text {nat }}$ and $\mathbf{J}^{\text {nat }}: \mathbb{R}^{m+N} \rightarrow$ $\mathbb{R}^{m+N}$ as follows.

$$
\mathbf{G}^{\text {nat }}(x, \Lambda):=\left(\begin{array}{c}
x_{1}-\Pi_{+}\left(x_{1}-\nabla_{1} \varphi_{1}(x)+\lambda_{1} \nabla_{1} c(x)\right) \\
\vdots \\
x_{N}-\Pi_{+}\left(x_{N}-\nabla_{N} \varphi_{N}(x)+\lambda_{N} \nabla_{N} c(x)\right) \\
\lambda_{1}-\Pi_{+}\left(\lambda_{1}-c(x)\right) \\
\vdots \\
\lambda_{N}-\Pi_{+}\left(\lambda_{N}-c(x)\right)
\end{array}\right), \mathbf{J}^{\text {nat }}(x, \Lambda)=\left(\begin{array}{c}
x_{1}-\Pi_{+}\left(x_{1}-\nabla_{1} \varphi_{1}(x)+\lambda_{1} \nabla_{1} c(x)\right) \\
\vdots \\
x_{N}-\Pi_{+}\left(x_{N}-\nabla_{N} \varphi_{N}(x)+\lambda_{N} \nabla_{N} c(x)\right) \\
\lambda_{1}-\Pi_{+}\left(\lambda_{1}-c(x)\right) \\
\lambda_{2}-\lambda_{1} \\
\vdots \\
\lambda_{N}-\lambda_{1}
\end{array}\right)
$$

$\forall x \in \mathbb{R}^{m}, \Lambda \in \mathbb{R}^{N}$, where $\Pi_{+}(\cdot)$ is the Euclidean projection on the nonnegative orthant of appropriate dimension. Recall that the relation $0 \leq u \perp v \geq 0$ is equivalent to $u=\Pi_{+}(u-v)$ [7]. It follows from comparison with $\mathrm{KKT}_{i}$ 's that $x$ solves $\mathrm{QVI}(K, F)$ if and only if there exists $\Lambda \in \mathbb{R}^{N}$ such that $\mathbf{G}^{\text {nat }}(x, \Lambda)=0$. By Theorem $15, x$ solves $\operatorname{VI}(\mathbb{C}, F)$ if and only if there exists $\Lambda \in \mathbb{R}^{N}$ such that $\mathbf{J}^{\text {nat }}(x, \Lambda)=0$. Notice the structure of $\mathbf{J}^{\text {nat }}$ : contrary to popular approaches to characterizing the shared multiplier that express the shared multiplier as a point in $\mathbb{R}$, we treat the shared multiplier explicitly as a vector in $\mathbb{R}^{N}$ with identical coordinates. For the result below, it is analytically convenient to have equation reformulations of the GNE and VE with the same domain $\left(\mathbb{R}^{m+N}\right)$.

Theorem 16 Let $\Omega$ be an open bounded set in $\mathbb{R}^{m+N}$ such that $0 \notin \mathbf{G}^{\text {nat }}(\partial \Omega)$. Then

$$
\operatorname{deg}\left(\mathbf{G}^{\text {nat }}, \Omega, 0\right)=\operatorname{deg}\left(\mathbf{J}^{n a t}, \Omega, 0\right) .
$$

Proof : Observe that since $0 \notin \mathbf{G}^{\text {nat }}(\partial \Omega), \mathbf{J}^{\text {nat }}$ is not zero on $\partial \Omega$ and $\operatorname{deg}\left(\mathbf{G}^{\text {nat }}, \Omega, 0\right)$ and $\operatorname{deg}\left(\mathbf{J}^{\text {nat }}, \Omega, 0\right)$ are well defined. We again will invoke the homotopy invariance of the Brouwer degree. Define $H$ : $[0,1] \times \bar{\Omega} \rightarrow \mathbb{R}^{m+N}$ as

$$
H(\bar{t}, \bar{x}, \bar{\Lambda})=\bar{t} \mathbf{G}^{\mathrm{nat}}(\bar{x}, \bar{\Lambda})+(1-\bar{t}) \mathbf{J}^{\mathrm{nat}}(\bar{x}, \bar{\Lambda}) \quad \forall \bar{t} \in[0,1],(\bar{x}, \bar{\Lambda}) \in \bar{\Omega} .
$$

We will show that $\operatorname{deg}(H(\bar{t}, \cdot), \Omega, 0)$ is well defined for each $\bar{t} \in[0,1]$ and then invoke its invariance with respect to $\bar{t}$ to conclude the claim. We know that this degree is well defined for $t \in\{0,1\}$. Assume that it is not so for some $t \in(0,1)$, i.e. assume that for some $t \in(0,1)$ and $(x, \Lambda) \in \partial \Omega, H(t, x, \Lambda)=0$. Therefore

$$
\begin{aligned}
& x_{i}-\Pi_{+}\left(x_{i}-\nabla_{i} \varphi_{i}(x)+\nabla_{i} c(x) \lambda_{i}\right)=0 \quad \forall i \in \mathcal{N}, \\
& \lambda_{1}-\Pi_{+}\left(\lambda_{1}-c(x)\right)=0 \\
& t \Pi_{+}\left(\lambda_{i}-c(x)\right)+(1-t) \lambda_{1}=\lambda_{i} . \quad \forall i \in \mathcal{N} \backslash\{1\} .
\end{aligned}
$$

It follows that $(x, \Lambda) \in \mathbb{R}_{+}^{m+N}$. From (14) it is clear that $0 \leq \lambda_{1} \perp c(x) \geq 0$. Pick an arbitrary $i \neq 1$. We will show that $0 \leq \lambda_{i} \perp c(x) \geq 0$ holds for this $i$. From (15) it follows that $\lambda_{i} \geq 0$. Since $\lambda_{i}$ is a real number, two cases arise: 
(a) If $\lambda_{i} \geq c(x)$, then $\Pi_{+}\left(\lambda_{i}-c(x)\right)=\lambda_{i}-c(x)$. So we get from (15)

$$
(1-t)\left(\lambda_{i}-\lambda_{1}\right)=-t c(x) \Longrightarrow(1-t) \lambda_{i} c(x)=-t c(x)^{2} \Longrightarrow \lambda_{i} c(x)=0,
$$

where the last implication is deduced by from noting that $c(x) \geq 0, \lambda_{i} \geq 0$ and $t>0$.

(b) On the othe hand if $\lambda_{i}<c(x)$,

$$
\lambda_{i}=(1-t) \lambda_{1} \Longrightarrow \lambda_{i} c(x)=0
$$

Hence in either case, $0 \leq \lambda_{i} \perp c(x) \geq 0$ or $\lambda_{i}=\Pi_{+}\left(\lambda_{i}-c(x)\right)$. and so from (15), $\lambda_{i}=\lambda_{1}$. As $i$ was arbitrary, we have $\lambda_{1}=\lambda_{2}=\cdots=\lambda_{N}$. But this means $\mathbf{J}^{\text {nat }}(x, \Lambda)=0$, a contradiction to our hypothesis.

So $0 \notin H(\bar{t}, \partial \Omega)$ for all $\bar{t}$ in $[0,1]$ and $\operatorname{deg}(H(\bar{t}, \cdot), \Omega, 0)$ is independent of $\bar{t}$. The claim follows.

Remark : The above theorem can be generalized for games where $c: \mathbb{R}^{m} \rightarrow \mathbb{R}^{n}, n>1$. This would merely require steps (a) and (b) above to repeated for each of the $n$ components of $c(x)$.

Theorem 16 plays the same role in the primal-dual space as Theorem 8 did in the primal space, insofar as studying the VE as a refinement of the GNE. By an argument analogous to that in Theorem 9 , the VE is a refinement for the class of games for which the existence of a GNE implies that we can find an $\Omega$ as in Theorem 16 so that $\operatorname{deg}\left(\mathbf{G}^{\text {nat }}, \Omega, 0\right) \neq 0$. In the remainder of this section is we will develop a condition that ensures this.

Let $\psi: \mathbb{R}^{m+N} \rightarrow \mathbb{R}^{m+N}$ be defined as follows

$$
\psi(x, \Lambda):=\left(\begin{array}{cc}
\nabla_{1} \varphi_{1}(x)-\lambda_{1} \nabla_{1} c(x) & \\
\vdots & \\
\nabla_{N} \varphi_{N}(x)-\lambda_{N} \nabla_{N} c(x) & \\
c(x) & (N \text { times }) \\
\vdots & \\
c(x) &
\end{array}\right)
$$

Observe that $\mathbf{G}^{\text {nat }}$ is the natural map of $\operatorname{VI}\left(\mathbb{R}_{+}^{m+N}, \psi\right): \mathbf{G}^{\text {nat }}(z)=z-\Pi_{+}(z-\psi(z))$. Moreover, notice that $\operatorname{VI}\left(\mathbb{R}^{m+N}, \psi\right)$ is a cartesian $V I$ in the sense of [7] over the following set

$$
\mathbb{R}_{+}^{m+N}=\prod_{k=1}^{2 N} \mathbb{R}_{+}^{\nu_{k}}, \quad \text { where } \nu_{k}= \begin{cases}m_{k} & k=1, \ldots, N \\ 1 & k=N+1 \ldots, 2 N .\end{cases}
$$

Suppose $\psi$ is a $\mathbf{P}_{0}$ function and $\operatorname{VI}\left(\mathbb{R}_{+}^{m+N}, \psi\right)$ has a bounded solution set. It can be shown that $\operatorname{deg}\left(\mathbf{G}^{\text {nat }}, \Omega, 0\right)= \pm 1$ for any any open bounded set $\Omega$ that contains $\operatorname{SOL}\left(\operatorname{VI}\left(\mathbb{R}_{+}^{m+N}, \psi\right)\right)[7$, section 3.6.1]. Combining with Theorem 16, we see that such a game also admits a VE. This is articulated in the following theorem. The definition of a $\mathbf{P}_{0}$ mapping is as follows. $\psi: \mathbb{R}_{+}^{m+N} \rightarrow \mathbb{R}^{m+N}$ is said to $\mathbf{P}_{0}$ on $\mathbb{R}_{+}^{m+N}$ partitioned as in (16) if for all $x, y \in \mathbb{R}^{m+N}$, there exists an index $i \in\{1, \ldots, 2 N\}$ such that and $x_{i} \neq y_{i}$ and $\left(\psi_{i}(x)-\psi_{i}(y)\right)^{T}\left(x_{i}-y_{i}\right) \geq 0$.

Theorem 17 Suppose $\psi$ is a $\mathbf{P}_{0}$ mapping and $\left(\mathbf{G}^{\text {nat }}\right)^{-1}(0)$ (if nonempty) is bounded, then the implication

$$
\left(\mathbf{G}^{n a t}\right)^{-1}(0) \neq \emptyset \Longrightarrow\left(\mathbf{J}^{n a t}\right)^{-1}(0) \neq \emptyset,
$$

holds and this game admits the VE as a refinement of the GNE. 
Note that $\psi$ being $\mathbf{P}_{0}$ is not sufficient for $\operatorname{VI}\left(\mathbb{R}_{+}^{m+N}, \psi\right)$ to have a solution and $\mathcal{G}$ to have a GNE. But if a GNE exists, and the set of GNEs is bounded (in the primal-dual space), then the above theorem shows that a VE also exists. Consequently, the game in the above result belongs to $\mathcal{S} \backslash \mathcal{S}_{2}^{\prime}$. A sufficient condition for the boundedness of $\mathrm{SOL}\left(\mathrm{VI}\left(\mathbb{R}_{+}^{m+N}, \psi\right)\right)$ can be seen Theorem 5.5.15 in [7].

We conclude this section by giving a sufficient condition for $\psi$ to be a $\mathbf{P}_{0}$ function. Recall that $\psi$ is $\mathbf{P}_{0}$ if its Jacobian, $\nabla \psi(z)$, is a $\mathbf{P}_{0}$ matrix for any $z$. Lemma 18 gives a sufficient condition for $\nabla \psi$ to be a $\mathbf{P}_{0}$ matrix. Assume that $c$ is twice continuously differentiable and let the Jacobian of $\psi$ be defined as

$$
\Psi:=\nabla \psi=\left(\begin{array}{cc}
\mathbf{H} & \mathbf{B} \\
\mathbf{C} & \mathbf{0}
\end{array}\right)
$$

where submatrices $\mathbf{H}, \mathbf{B}, \mathbf{C}, \mathbf{0}$ are as indicated below.

$$
\Psi(x, \Lambda)=\left[\begin{array}{ccc|ccc}
\nabla_{11} \varphi_{1}(x)-\nabla_{11} c(x) \lambda_{1} & \cdots & \nabla_{1 N} \varphi_{1}(x)-\nabla_{1 N} c(x) \lambda_{1} & -\nabla_{1} c(x) & \cdots & 0 \\
& \vdots[\mathbf{H}] & & 0 & \ddots[\mathbf{B}] & 0 \\
\nabla_{N 1} \varphi_{N}(x)-\nabla_{1 N} c(x) \lambda_{N} & \cdots & \nabla_{N N} \varphi_{N}(x)-\nabla_{N N} c(x) \lambda_{N} & 0 & \cdots & -\nabla_{N} c(x) \\
\hline \nabla_{1} c(x)^{T} & \cdots & \nabla_{N} c(x)^{T} & 0 & \cdots & 0 \\
\vdots & \vdots[\mathbf{C}] & \vdots & 0 & \ddots[\mathbf{0}] & 0 \\
\nabla_{1} c(x)^{T} & \cdots & \nabla_{N} c(x)^{T} & 0 & \cdots & 0
\end{array}\right] .
$$

Lemma 18 Let $c: \mathbb{R}^{m} \rightarrow \mathbb{R}$ be a concave function in $C^{2}$. Assuume that for all $x, \Lambda \geq 0 \mathbf{H}(x, \Lambda)$ is a block diagonal positive definite matrix with blocks $\mathbf{H}_{1,1}, \ldots, \mathbf{H}_{N, N}$ where for each $i \in \mathcal{N}$, the submatrix $\mathbf{H}_{i, i}$ is a positive definite matrix in $\mathbb{R}^{m_{i} \times m_{i}}$. Then $\Psi(x, \Lambda)$ is a $\mathbf{P}_{0}$ matrix.

Proof : The proof follows by showing that every principal submatrix of $\Psi(x, \Lambda)$ has a nonnegative determinant. Consider an arbitrary submatrix $D$ is given by $D=\Psi(x, \Lambda)_{\alpha, \alpha}$ where $\alpha \subseteq\{1, \ldots, m+N\}$ is an index set. Let $\alpha=\beta \cup \gamma$, where $\beta \subseteq\{1, \ldots, m\}, \gamma \subseteq\{m+1, \ldots, m+N\}$. Then $\Psi(x, \Lambda)_{\alpha, \alpha}$ is given by

$$
\left(\begin{array}{cc}
\mathbf{H}(x, \Lambda)_{\beta, \beta} & \mathbf{B}(x)_{\beta, \gamma} \\
\mathbf{C}(x)_{\gamma, \beta} & \mathbf{0}_{\gamma, \gamma}
\end{array}\right)
$$

We drop arguments $(x)$ and $(x, \Lambda)$ for brevity. Consider some $\beta \subseteq\{1, \ldots, m\}$. If $\left[\mathbf{C}_{\gamma, \beta}, \mathbf{0}_{\gamma, \gamma}\right]$, has at least 2 identical rows it follows that $\operatorname{det}(D)=0$. Since $c$ is $\mathbb{R}$-valued, it suffices to consider the case where $|\gamma| \leq 1$ and $\left[\mathbf{C}_{\gamma, \beta}, \mathbf{0}_{\gamma, \gamma}\right]$ does not contain all zeros. For any $i \in \beta$ let $b_{i}:=\min \left\{k \mid i \leq \sum_{j=1}^{k} m_{j}\right\}$ and let $\kappa_{i}=m+b_{i}$. For a row $i \in \beta, \kappa_{i}$ is the column of $\mathbf{B}$ that contains the vector $-\nabla_{j} c$ through which row $i$ passes. i.e. if $\mathbf{B}[i, j]$ denotes the element in the $i^{\text {th }}$ row and $j-m^{\text {th }}$ column of $\mathbf{B}$, we have,

$$
j \neq \kappa_{i} \Longrightarrow \mathbf{B}[i, j]=0 \text {. }
$$

Recall that $\gamma$ has at most one element. Based on $\gamma$ three cases arise:

1. $\gamma=\emptyset$ : In this case $D$ is a principal submatrix of $\mathbf{H}$. Since $\mathbf{H} \succ 0$, it follows that $\operatorname{det}(D) \geq 0$.

2. $\gamma \neq \emptyset, \gamma \cap \bigcup_{i \in \beta} \kappa_{i}=\emptyset$ : Let $\gamma=\{j\}$. This assumption ensures that for all $i \in \beta, j \neq \kappa_{i}$. Hence there is a column of $\mathbf{B}_{\beta, \gamma}$ that has all zeros. Consequently there is a zero column of $D$. Hence $\operatorname{det}(D)=0$. 
3. $\gamma \neq \emptyset, \gamma \cap \bigcup_{i \in \beta} \kappa_{i}=\gamma$ : Let $\gamma=\{j\}$ This means that there is an $i \in \beta$ such that $j=\kappa_{i}$. Assume that $j=m+1$ and $\beta=\{1, \ldots, m\}$. We shall see that there is no loss of generality in this assumption. Then, recalling that $\mathbf{H}$ is block diagonal, $D$ may be written as

$$
D=\left(\begin{array}{cccc}
\mathbf{H}_{1,1} & \ldots & \mathbf{0}_{1, N} & -\nabla_{1} c \\
& \ddots & & 0 \\
\mathbf{0}_{N, 1} & \ldots & \mathbf{H}_{N, N} & 0 \\
\nabla_{1} c^{T} & \ldots & \nabla_{N} c^{T} & 0
\end{array}\right)
$$

where $\mathbf{H}_{i, i} \in \mathbb{R}^{m_{i} \times m_{i}}$. Using the Schur complement [13] we may write the determinant of $D$ as

$$
\begin{aligned}
\operatorname{det}(D) & =\operatorname{det}(\mathbf{H}) \operatorname{det}\left(-\mathbf{C}_{\gamma, \beta} \mathbf{H}^{-1} \mathbf{B}_{\beta, \gamma}\right) \\
& =\operatorname{det}(\mathbf{H}) \operatorname{det}\left(\nabla_{1} c^{T} \mathbf{H}_{1,1}^{-1} \nabla_{1} c\right) \geq 0 .
\end{aligned}
$$

Since $c$ is $\mathbb{R}$-valued, $\nabla_{1} c$ is a vector. The nonnegativity of $\operatorname{det}(D)$ follows from the positive definiteness of $\mathbf{H}$ and of the inverse of $\mathbf{H}_{1,1}$.

It is easy to see that the above arguments would hold if we picked $\gamma$ to comprise some other element $(\neq m+1)$ and $\mathbf{H}_{\beta, \beta}$ was any other principal submatrix of $\mathbf{H}$.

Remark : The above result has assumed that $c$ is $\mathbb{R}$-valued. Extending this result to higher dimensions may require making stronger assumptions on the properties of the Jacobian, so as to ensure that the resulting Schur complement is a $\mathbf{P}_{0}$ matrix.

An example of a game where the hypotheses of Lemma 18 are satisfied is network routing game with affine coupling constraints considered in [14]. We present a modification of this game below.

Example 5. A network routing game: Assume each player has real valued strategies and solves an optimization problem given by

\begin{tabular}{|cc|}
$\mathrm{A}_{i}\left(x^{-i}\right) \quad \operatorname{minimize}_{x_{i}}$ & $\varphi_{i}\left(x_{i} ; x^{-i}\right)=\mathcal{U}_{i}\left(x_{i}\right)$ \\
& $a^{T} x \geq b \quad: \lambda_{i}$ \\
subject to & $x \geq 0$, \\
\hline
\end{tabular}

where $a \in \mathbb{R}^{m}, b \in \mathbb{R}$ and $\mathcal{U}_{i}$ is a convex continuously differentiable function. This is clearly a shared constraint game with $m_{i}=1$ for all $i \in \mathcal{N}$ and $\mathbb{C}=\left\{x \mid a^{T} x \geq b\right\}$. If $\mathcal{U}_{i}$ are strictly convex for each $i \in \mathcal{N}, F$ is a strictly monotone function and $\mathbf{H}$ is a positive definite diagonal matrix. Ordinarily, this would not be sufficient to claim that $\mathrm{VI}(\mathbb{C}, F)$ has a solution and that this game has a VE. But if given that this game has a bounded and nonempty set of GNEs (in the primal-dual space), one may use Theorem 16 and ?? and Lemma 18 to conclude that a VE exists for this game. In other words, for this game, the VE is a refinement of the GNE and this game belongs to the class $\mathcal{S} \backslash \mathcal{S}_{2}^{\prime}$. In fact, using the strict monotonicity of $F$, we may also claim that the VE is unique. Furthermore, if we are independently given the boundedness of the (possibly empty) set of GNEs in the primal-dual space, we may say that the game either admits a VE or admits no GNE at all. This is also compatible with economic intuition that for such games, the equilibrium with uniform prices is the more appealing solution concept and is the one applied in [14]. This is another example where our results provide a unification of ideas that were either previously known or had an intuitive appeal, but no formal justification. 


\section{Refinement of the generalized Nash equilibrium in power markets}

In this section, we show that a shared-constraint generalized Nash game arising in power markets, admits the VE as a refinement of its GNE. The model is fairly broad and may also be applied to a game of capacity expansion [17] with demand constraints. The game is distinguished by its model which depicts Cournot competition. Since the constraint of serving demand is "shared" by all players, this game is a generalized Nash game with shared constraints.

Consider a power market comprising of firms $\mathcal{N}=\{1, \ldots, N\}$ competing over an electricity network comprising of a set of nodes, denoted by $\mathbf{N}$. The $j^{\text {th }}$ firm may own generation facilities at a subset of nodes denoted by $\mathbf{N}_{j}$ and we denote the set of firms with such facilities at node $i$ by $\mathcal{N}_{i}$. The objective of generating firms is to choose their generation level so as to maximize their profit subject to the sum of their generations being sufficient to meet the demand.

In the market we assume, the price of electricty at the nodes is determined via a Cournot model, i.e. the price is blind to the generation levels of individual generators present at the node and is dependent only on the sum of their generations. Specifically, if $\widehat{x}_{i}:=\left(x_{j i}\right)_{j \in \mathcal{N}_{i}}$ are the generation levels at node $i$, $p_{i}$, the price at node $i$, is given by

$$
p_{i}\left(\widehat{x}_{i}\right):=a_{i}-b_{i} \sum_{j \in \mathcal{N}_{i}} x_{j i}, \quad \forall i \in \mathbf{N} .
$$

Finally, let the cost of generation of quantity $x_{j i}$ for firm $j$ at node $i$ be $\zeta_{j i}\left(x_{j i}\right)$, whereby the loss, $\varphi_{j}$ of firm $j$ is given by :

$$
\varphi_{j}\left(x_{j} ; x^{-j}\right):=-\sum_{i \in \mathbf{N}_{j}}\left(p_{i}\left(\widehat{x}_{i}\right) x_{j i}-\zeta_{j i}\left(x_{j i}\right)\right),
$$

where $x_{j}$ is defined as $x_{j}:=\left(x_{j i}\right)_{i \in \mathbf{N}_{j}}$. Unlike before, we follow a special convention in denoting the tuple of strategies of all players. Let $n=|\mathbf{N}|$ be the number of nodes in the network. The tuple of strategies $x$ is denoted by $\left(\widehat{x}_{1}, \ldots, \widehat{x}_{n}\right)$ and for a firm $j \in \mathcal{N}$, we denote the tuple $x^{-j}:=\left(\widehat{x}_{1}^{-j}, \ldots, \widehat{x}_{n}^{-j}\right)$ where $\widehat{x}_{i}^{-j}:=\left(x_{k i}\right)_{k \in \mathcal{N}_{i} \backslash j}$. If $d_{i}$ denotes the demand at node $i$, the shared constraint that the firms' generations are required to satisfy is

$$
\mathbb{C}:=\left\{x \mid x \geq 0, \sum_{j \in \mathcal{N}_{i}} x_{j i} \geq d_{i}, \forall i \in \mathbf{N}\right\} .
$$

As before we denote the feasible region of generation firm $j$ 's optimization problem as

$$
K_{j}\left(z^{-j}\right):=\left\{z \mid\left(z, z^{-j}\right) \in \mathbb{C}\right\}, \quad \forall j \in \mathcal{N},
$$

and let $K=\prod_{j \in \mathcal{N}} K_{j}$. The resulting maximization problem faced by the $j^{\text {th }}$ firm is given by

$$
\begin{array}{|lll|}
\hline \mathrm{A}_{j}\left(x^{-j}\right) & \begin{array}{l}
\operatorname{minimize}_{x_{j}} \\
\text { subject to }
\end{array} & \varphi_{j}\left(x_{j} ; x^{-j}\right) \\
&
\end{array}
$$

The game resulting out of problems $\mathrm{A}_{j}$ is clearly a generalized Nash game with shared constraints. The GNE of this game is often referred to as the Nash-Cournot equilibrium, with an additional demand constraint. The variational equilibrium $(\mathrm{VE})$ of this game is given by the solution to $\mathrm{VI}(\mathbb{C}, F)$ where $F:=\left(\left(F_{1}\right)^{T}, \quad \ldots,\left(F_{n}\right)^{T}\right)^{T}$, where

$$
F_{i}\left(\widehat{x}_{i}\right)=\left[\frac{\partial \varphi_{j}\left(x_{j} ; x^{-j}\right)}{\partial x_{j i}}\right]_{j \in \mathcal{N}_{i}} .
$$


Proposition 19 Let $x^{\text {ref }} \in \partial \mathbb{C}$ be a GNE of the above game with $F\left(x^{r e f}\right) \in-\operatorname{int}\left(\mathscr{N}\left(x^{r e f} ; K\left(x^{r e f}\right)\right)\right.$. If the generation cost $\zeta_{j i}$ are convex for all $j \in \mathcal{N}, i \in \mathbf{N}$ and then the game $\mathcal{G}$ admits a VE.

Proof : Let $m_{i}=\left|\mathcal{N}_{i}\right|$ be the number of firms with generation at node $i$ and $m=\sum_{i \in \mathbf{N}} m_{i}$. Thus the tuple of the strategies of all firms is a vector in $\mathbb{R}^{m}$. We begin by observing that $\mathbb{C}=\left\{x \in \mathbb{R}^{m} \mid x \geq\right.$ $0, A x \geq d\}$, where $A$ is a $n \times m$ matrix, $d$ is a $n$-dimensional vector of demands, $\left[d_{1}, \ldots, d_{n}\right]$. Every element of $A$ is either 0 or 1 , according to the following rule:

$$
A[i, j]= \begin{cases}1 & \text { if } \quad \sum_{k=0}^{i-1} m_{k}<j \leq \sum_{k=0}^{i} m_{k} \\ 0 & \text { else }\end{cases}
$$

where we define $m_{0}:=0$. By the same argument as in Example 3 , we get $\mathbb{C}_{\infty}=\mathscr{T}\left(x^{\text {ref }} ; K\left(x^{\text {ref }}\right)\right)$, whereby condition (2) from Theorem 14 holds. And since $F\left(x^{\mathrm{ref}}\right) \in-\operatorname{int}\left(\mathscr{N}\left(x^{\mathrm{ref}} ; K\left(x^{\mathrm{ref}}\right)\right)\right)$, condition (1) from Theorem 14 also holds. To show the result, it remains to show that $F$ is pseudo-monotone, which holds if $F$ is monotone. From above,

$$
F_{i}\left(\widehat{x}_{i}\right)=-\left(p_{i}\left(\widehat{x}_{i}\right)-b_{i} x_{j i}-\zeta_{j i}^{\prime}\left(x_{j i}\right)\right)_{j \in \mathcal{N}_{i}},
$$

and therefore

$$
\nabla F_{i}\left(\widehat{x}_{i}\right):=\operatorname{diag}\left[b_{i}+\zeta_{j i}^{\prime \prime}\left(x_{j i}\right)\right]_{j \in \mathcal{N}_{i}}+b_{i} E_{i},
$$

where $E_{i}=e_{i} e_{i}^{T}$ and $e_{i}$ an $m_{i}$-dimensional vector with each element 1 . Since $\varphi_{j}$ 's are nodally separable, we get $\nabla F=\operatorname{diag}\left[\nabla F_{i}\right]_{i \in \mathcal{N}}$. It is now easy to see that $\nabla F$ is positive semidefinite. Observe that

$$
s^{T} E_{i} s=s^{T} e_{i} e_{i}^{T} s=\left(\sum s_{j}\right)^{2} \geq 0,
$$

and consequently $E_{i}$ is a positive semidefinite matrix. Since $\zeta_{j i}$ 's are convex and $b_{i}$ is nonnegative, $\nabla F_{i}$ is a sum of two positive semidefinite matrices, from which the result follows.

\subsection{Some more examples of shared constraint games}

In this section, we construct examples that illustrate the strange properties generalized Nash games with shared constraints can exhibit. Often these properties are contributed by the structure of the set-valued map $K$, particularly on points on the boundary of the domain of $K$. Recall Theorem 8 where we showed that $\operatorname{deg}\left(\widetilde{\mathbf{F}}_{K}^{\text {nat }}, \Omega, 0\right)=\operatorname{deg}\left(\mathbf{F}_{\mathbb{C}}^{\text {nat }}, \Omega, 0\right)$, where $\Omega$ was an open bounded set inside $\operatorname{dom}(K)$. Theorem 8 applies only when $\Omega$ is taken from the interior of $\operatorname{dom}(K)$. We will see in the examples below that on the boundary of $\operatorname{dom}(K), \mathrm{QVI}(K, F)$ can show surprising behaviour. Throughout we assume $N=2$ and $m_{1}=m_{2}=1$.

Example 6. Game with GNE independent of $F$ : Often the nature of $K$ (and $\mathbb{C}$ ) plays a dominant role in determining $\operatorname{SOL}(\mathrm{QVI}(K, F))$, in the sense that it renders some points as solutions regardless of $F$. Fig. 2 shows $\mathbb{C}$ and a point $x^{*} \in \partial \mathbb{C}$ with the property that for any $F, x^{*}$ solves $\mathrm{QVI}(K, F)$. This is because the image of $x^{*}$ under $K$ is a singleton, namely $x^{*}$ itself. In Fig. 2, dotted lines depict axes with their origin shifted to $x^{*}$. If $y \in K\left(x^{*}\right)$, the points $\left(y_{1}, x_{2}^{*}\right)$ and $\left(x_{1}^{*}, y_{2}\right)$ lie in on these 'axes'. Notice that since these 'axes' intersect $\mathbb{C}$ at only one point, $x^{*}, K\left(x^{*}\right)=\left\{x^{*}\right\}$. As a result for any $F$, we have

$$
F\left(x^{*}\right)^{T}\left(y-x^{*}\right)=0 \quad \forall y \in K\left(x^{*}\right)
$$

implying that $x^{*} \in \operatorname{SOL}(\mathrm{QVI}(K, F))$. Observe that $x^{*}$ lies in $\partial \operatorname{dom}(K)$. 


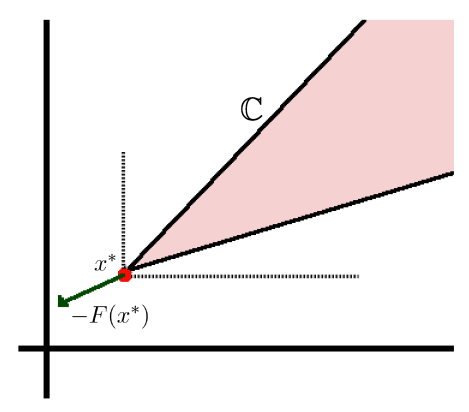

Figure 2: An example where $K\left(x^{*}\right)=\left\{x^{*}\right\}$. Independently of $F, x^{*}$ solves QVI $(K, F)$. When $x^{*}$ is the origin and $F(x)=\left(x_{1}, 1\right), x^{*}$ is the only solution.

Example 7. Game with unique GNE and VE: While sufficiency conditions for a manifold of solutions to a QVI were provided in Theorem 20, QVIs with unique solutions also exist. Suppose $x^{*}$ in Fig. 2 is the origin of the coordinate system, i.e. let $x^{*}=(0,0)$. Assume that $\mathbb{C}=\left\{\left(x_{1}, x_{2}\right) \mid x \geq\right.$ $\left.0, x_{1} \in\left[x_{2}, 2 x_{2}\right]\right\}$, so that

$$
K(x)=\left\{\left(y_{1}, y_{2}\right) \mid y \geq 0, y_{1} \in\left[x_{2}, 2 x_{2}\right], y_{2} \leq x_{1} \leq 2 y_{2}, x \geq 0\right\} .
$$

Let $F(x)=\left(x_{1}, 1\right)$ (arising from, say, $\left.\varphi_{1}(x)=\frac{1}{2} x_{1}^{2}+x_{2}, \varphi_{2}(x)=x_{2}-x_{1}\right)$. This game has a unique GNE, $x^{*}$. Indeed one may verify that $K\left(x^{*}\right)=\left\{x^{*}\right\}$, and conclude from the previous example that $x^{*}$ is a solution for any $F$. To see that this is the only solution, suppose $x \in \mathbb{C} \backslash\left\{x^{*}\right\}$ solves $\mathrm{QVI}(K, F)$. i.e.

$$
\left(\begin{array}{c}
x_{1} \\
1
\end{array}\right)^{T}\left[\left(\begin{array}{l}
y_{1} \\
y_{2}
\end{array}\right)-\left(\begin{array}{l}
x_{1} \\
x_{2}
\end{array}\right)\right] \geq 0 \quad \forall y \in K(x) .
$$

Observe that $y=\left(x_{1}, \frac{1}{2} x_{1}\right) \in K(x)$ since $x_{1} \in\left[x_{2}, 2 x_{2}\right]$. Substituting above gives $x_{1} \geq 2 x_{2}$, which implies the solution must satisfy $x_{1}=2 x_{2}$. Now observe that $y=\left(x_{2}, \frac{1}{2} x_{1}\right)=\left(\frac{1}{2} x_{1}, \frac{1}{2} x_{1}\right) \in K(x)$. Substituting this $y$ above gives $\frac{1}{2} x_{1}^{2} \leq 0$. It follows that $x=(0,0)=x^{*}$, a contradiction. That makes $x^{*}$ the only solution. Notice that Theorem 20 does not apply here because $\left(x^{*}, \Lambda^{*}\right) \ngtr 0$. It is easy to check that $x^{*}$ also solves $\mathrm{VI}(\mathbb{C}, F)$, i.e. $x^{*}$ is a $\mathrm{VE}$, and is the unique VE. Thus, for this game, $\mathrm{SOL}(\mathrm{VI}(\mathbb{C}, F))$ $=\operatorname{SOL}(\mathrm{QVI}(K, F))$.

\section{Conclusions}

In this paper, we presented a theory of the VE as a refinement of the GNE. The GNE and the VE were shown to related via a Brouwer degree-theoretic equivalence in the primal and primal-dual space. These equivalences led to sufficiency conditions for a shared constraint game to have the VE as a refinement of the GNE. Furthermore, for certain games these conditions were seen as necessary. Finally, this framework was applied on a class of Nash-Cournot games in power market games where it was shown that the VE was indeed a refinement of the GNE.

\section{A Appendix}

\section{A.1 On the existence of a manifold of GNEs}

A remark made in [5] claimed that generalized Nash games often have a manifold of equilibria. In Example 2, we saw a QVI with a unique solution. Theorem 20 below shows that manifolds may exist 
under some conditions on the dimensions of the QVI. Here we assume $c: \mathbb{R}^{m} \rightarrow \mathbb{R}^{n}$ to be a $C^{1}$ concave function and that an appropriate constraint qualification holds.

Theorem 20 Consider a game in which $m=n N$. Let $\left(x^{*}, \Lambda^{*}\right)>0$ be a GNE of such a game such that the square matrix $\mathbf{B}\left(x^{*}\right)$ is nonsingular. Then there exists a neighbourhood $B\left(x^{*}, r\right) \subseteq \mathbb{R}^{m}$ of $x^{*}$ of radius $r$ such that for every $x \in B\left(x^{*}, r\right) \cap\{v \mid c(v)=0\}$, there exists $\Lambda \geq 0$ so that $(x, \Lambda)$ is a GNE.

Proof : Since $\left(x^{*}, \Lambda^{*}\right)>0$ it is easy to see from the KKT conditions and nonsingularity of $\mathbf{B}\left(x^{*}\right)$ that

$$
\Lambda^{*}=-\mathbf{B}\left(x^{*}\right)^{-1} F\left(x^{*}\right) .
$$

$\operatorname{det}(\mathbf{B}(\cdot))$ is a $\mathbb{R}^{m} \rightarrow \mathbb{R}$ continuous function. By continuity there is a neighbourhood $B\left(x^{*}, r_{1}\right) \subseteq \mathbb{R}^{m}$ of $x^{*}$ such that sgn $\operatorname{det}(\mathbf{B}(x))=\operatorname{sgn} \operatorname{det}\left(\mathbf{B}\left(x^{*}\right)\right)$ for all $x \in B\left(x^{*}, r_{1}\right)$. Thus $\mathbf{B}(x)$ is nonsingular on $B\left(x^{*}, r_{1}\right)$. Furthermore since $-\mathbf{B}\left(x^{*}\right)^{-1} F\left(x^{*}\right)>0$ and $x^{*}>0$ there is another neighbourhood $B\left(x^{*}, r_{2}\right)$ of $x^{*}$ such that for all $x \in B\left(x^{*}, r_{2}\right),-\mathbf{B}(x)^{-1} F(x) \geq 0$ and $x>0$.

Finally, let $r=\min \left\{r_{1}, r_{2}\right\}$ and pick an arbitrary $x \in B\left(x^{*}, r\right) \cap\{v \mid c(v)=0\}$. Since $r \leq r_{2}, x>0$. Using this it is easy to see that for this $x$, the pair $(x, \Lambda)$, where $\Lambda=-\mathbf{B}(x)^{-1} F(x)$, is a GNE.

Remark : Theorem 20 can be extended to the case where $m<n N$ by replacing the hypothesis of nonsingularity of $\mathbf{B}\left(x^{*}\right)$ with one of full row-rank.

\section{A.2 Proof of Lemma 5}

\section{Proof :}

1. Take any $i \in \mathcal{N}$ and consider an $x \in \mathbb{R}^{m}$. Note from (1) and the cartesian nature assumed on $\mathbb{C}$ that $K_{i}\left(x^{-i}\right)=\left\{y_{i} \in \mathbb{R}^{m_{i}} \mid\left(y_{i}, x^{-i}\right) \in \mathbb{C}\right\}=\left\{y_{i} \in \mathbb{R}^{m_{i}} \mid y_{i} \in \mathbb{C}_{i}, x_{j} \in \mathbb{C}_{j}, j \neq i\right\}$, which is nonempty if $x_{j} \in \mathbb{C}_{j}, \forall j \neq i$. Thus $K(x)=\prod K_{i}\left(x^{-i}\right) \neq \emptyset$ if and only if $x \in \mathbb{C}$. Similarly, for $x \in \mathbb{C}$, we have $y \in K(x)$ if and only if $y \in \mathbb{C}$. Therefore $K(x)=\mathbb{C}$ if and only if $x \in \mathbb{C}$.

2. Let $x \in K(x)$ implying that $x_{i} \in K_{i}\left(x^{-i}\right), \forall i \in \mathcal{N}$, and therefore $\left(x_{i}, x^{-i}\right) \in \mathbb{C}, \forall i \in \mathcal{N}$ and $x \in \mathbb{C}$. The converse follows by noting that $x \in \mathbb{C}$ is equivalent to $\left(x_{i}, x^{-i}\right) \in \mathbb{C} \forall i$, i.e. $x_{i} \in K_{i}\left(x^{-i}\right), \forall i$ and therefore $x \in K(x)$.

3. Let $x \in \operatorname{dom}(K)$ and $y, z \in K(x)$, i.e. for each $i \in \mathcal{N},\left(y_{i}, x^{-i}\right)$ and $\left(z_{i}, x^{-i}\right) \in \mathbb{C}$. The convexity of $K(x)$ follows by noting that since $\mathbb{C}$ is convex, $\left(\left(\alpha y_{i}+(1-\alpha) z_{i}\right), x^{-i}\right) \in \mathbb{C}$ for each $i$ and $\alpha \in[0,1]$.

To show closedness, consider a sequence $\left\{y^{k}\right\} \subseteq K(x)$ with limit point $\bar{y}$. By closedness of $\mathbb{C}$, for each $i$, the sequence $\left\{\left(y_{i}^{k}, x^{-i}\right)\right\} \subseteq \mathbb{C}$ and $\lim \left(y_{i}^{k}, x^{-i}\right)=\left(\bar{y}_{i}, x^{-i}\right) \in \mathbb{C}$. Thus $K(x)$ is closed.

4. Suppose $x$ is a point in $\mathbb{C}, d \in K(x)_{\infty}$ is an arbitrary recession direction and $\tau$ is an arbitrary nonnegative number. By convexity of $\mathbb{C}$, it suffices to show that $x+\tau d \in \mathbb{C}$. Since $d \in K(x)_{\infty}$, and $x \in K(x)$, the point $x+N \tau d$ belongs to $K(x)$. Therefore the points $z^{i}:=\left(x_{i}+N \tau d_{i}, x^{-i}\right), i \in \mathcal{N}$, belong to $\mathbb{C}$. By convexity of $\mathbb{C}$, the average of these points

$$
\frac{1}{N} \sum_{i \in \mathcal{N}} z^{i}=\frac{N-1}{N} x+\frac{1}{N}(x+N \tau d)=x+\tau d,
$$

also belongs to $\mathbb{C}$, as required. If $\mathbb{C}$ is bounded, $\mathbb{C}_{\infty}=\{0\}$ and we get $K(x)_{\infty} \subseteq\{0\}$. Therefore $K(x)_{\infty}=\{0\}$, implying that $K(x)$ is bounded. 


\section{A.3 Proof of Lemma 6}

Proof : Suppose $x \in \operatorname{int}(\mathbb{C})$. Then there exist open sets $\mathcal{O}_{i} \subseteq \mathbb{R}^{m_{i}}$ containing $x_{i}$ such that $x \in \mathcal{O}:=$ $\prod_{i \in \mathcal{N}} \mathcal{O}_{i} \subseteq \mathbb{C}$. Then $\left(\mathcal{O}_{i}, x^{-i}\right):=\cup_{y_{i} \in \mathcal{O}_{i}}\left(y_{i}, x^{-i}\right) \subseteq \mathbb{C}$, so that $\mathcal{O}_{i} \subseteq K_{i}\left(x^{-i}\right)$, for each $i \in \mathcal{N}$. It follows that $\mathcal{O} \subseteq K(x)$.

For the converse, let int $(K(x))$ be nonempty and $x \in \operatorname{int}(K(x))$. Then for each $i \in \mathcal{N}, x_{i}$ belongs the interior of $K_{i}\left(x^{-i}\right)$ (where $K_{i}\left(x^{-i}\right)$ is considered a set in $\mathbb{R}^{m_{i}}$ ). Thus there exist open sets $\mathbb{R}^{m_{i}} \supseteq$ $\mathcal{O}_{i} \subseteq K_{i}\left(x^{-i}\right)$ containing $x_{i}$ for all $i$. It follows that $\left(\mathcal{O}_{i}, x^{-i}\right) \subseteq \mathbb{C}$ for all $i \in \mathcal{N}$. Now since $\mathbb{C}$ is convex, the average of these sets is contained in $\mathbb{C}$, i.e.

$$
\mathcal{A}:=\sum_{i \in \mathcal{N}} \frac{\left(\mathcal{O}_{i}, x^{-i}\right)}{N}=\frac{1}{N} \prod_{i \in \mathcal{N}} \mathcal{O}_{i}+\frac{N-1}{N} x \subseteq \mathbb{C} .
$$

Since $x_{i} \in \mathcal{O}_{i}, \mathcal{A}$ contains $x$. Furthermore, $\mathcal{A}$ is open, implying that $x \in \operatorname{int}(\mathbb{C})$.

\section{A.4 Cones}

For any closed convex set $S$, by $S_{\infty}$ we denote its recession cone [7, 23, page 158]:

$$
S_{\infty}=\{d \mid S+\tau d \subseteq S, \forall \tau \geq 0\}
$$

For any set $T$ and a point $z \in T$, let $\mathscr{N}(z ; T), \mathscr{T}(z ; T)$ denote the normal cone and the tangent cone of $T$ at $z$ respectively.

$$
\begin{aligned}
\mathscr{N}(z ; T) & =\left\{d \mid d^{T}(y-z) \leq 0 \quad \forall y \in T\right\} \\
\text { and } \quad \mathscr{T}(z ; T) & =\left\{d \mid \exists\left\{\tau_{k}\right\} \subseteq(0, \infty) \text { and }\left\{y_{k}\right\} \subseteq T, \text { s.t. } \tau_{k} \rightarrow 0, y_{k} \rightarrow z, \text { and } d=\lim _{k \rightarrow \infty} \frac{y_{k}-x}{\tau_{k}}\right\} .
\end{aligned}
$$

For any cone $T$, we use $T^{*}$ to denote its dual cone as $T^{*}=\left\{d \mid d^{T} x \geq 0 \forall x \in T\right\}$.

\section{References}

[1] K. Arrow and G. Debreu, Existence of an equilibrium for a competitive economy, Econometrica, 22 (1954), pp. 290, 265.

[2] J.-P. Aubin and H. Frankowska, Set-valued Analysis, Springer, 1990.

[3] T. Başar and G. Olsder, Dynamic Noncooperative Game Theory, Classics in Applied Mathematics, SIAM, Philadelphia, 1999.

[4] G. Debreu, A Social Equilibrium Existence Theorem, Proceedings of the National Academy of Sciences, 38 (1952), pp. 886-893.

[5] F. Facchinei, A. Fischer, And V. Piccialli, On generalized Nash games and variational inequalities, Operations Research Letters, 35 (2007), pp. 159-164.

[6] F. Facchinei And C. Kanzow, Generalized Nash equilibrium problems, 4OR: A Quarterly Journal of Operations Research, 5 (2007), pp. 173-210.

[7] F. FaCchinei And J.-S. PAng, Finite-Dimensional Variational Inequalities and Complementarity Problems I, Springer, 1 ed., Feb. 2003. 
[8] F. Facchinei and J.-S. PAng, Nash Equilibria: The Variational Approach, Convex Optimization in Signal Processing and Communication, Cambridge University Press, 2009.

[9] I. Fonsech and W. Gangbo, Degree Theory in Analysis and Applications, Clarendon Press, Apr. 1995.

[10] M. S. GowdA, Applications of degree theory to linear complementarity problems, Math. Oper. Res., 18 (1993), pp. 868-879.

[11] M. S. Gowda AND J.-S. PANG, Stability analysis of variational inequalities and nonlinear complementarity problems, via the mixed linear complementarity problem and degree theory, Math. Oper. Res., 19 (1994), pp. 831-879.

[12] P. T. HARKer, Generalized Nash games and quasi-variational inequalities, European Journal of Operational Research, 54 (1991), pp. 81-94.

[13] R. A. Horn and C. R. Johnson, Matrix analysis, Cambridge University Press, 1990.

[14] R. JohaRi AND J. N. Tsitsiklis, Efficiency loss in a newtork resource allocation game, Math. Oper. Res., 29 (2004), pp. 407-435.

[15] S. Kesavan, Nonlinear Functional Analysis: A First Course, Hindustan Book Agency, 2004.

[16] S. Leyffer And T. Munson, Solving multi-leader-follower games, Preprint ANL/MCS-P12430405, Argonne National Laboratory, Mathematics and Computer Science Division, (April, 2005).

[17] F. H. MurPhy And Y. Smeers, Generation capacity expansion in imperfectly competitive restructured electricity markets, Operations research, 53 (2003), pp. 646-661.

[18] R. B. Myerson, Refinements of the nash equilibrium concept, International Journal of Game Theory, 7 (1978), pp. $73-80$.

[19] R. B. Myenson, Game Theory: Analysis of Conflict, Harvard University Press, Sept. 1997.

[20] N. Nisan, T. Roughgarden, E. Tardos, and V. V. Vazirani, Algorithmic Game Theory, Cambridge University Press, Sept. 2007.

[21] D. O'Regan, Y. J. Cho, And Y.-Q. Chen, Topological Degree Theory and Applications, CRC Press LLC, 1 ed., Mar. 2006.

[22] J.-S. PAng And M. Fukushima, Quasi-variational inequalities, generalized nash equilibria, and multi-leader-follower games, Computational Management Science, 2 (2005), pp. 21-56.

[23] R. Rockafellar, Convex analysis, Princeton University Press, Princeton, NJ, 1997. Reprint of the 1970 original, Princeton Paperbacks.

[24] R. T. Rockafellar and R. J. Wets, Variational Analysis, Springer, Aug. 2009.

[25] J. B. Rosen, Existence and uniqueness of equilibrium points for concave n-person games, Econometrica, 33 (1965), pp. 520-534.

[26] R. Selten, Reexamination of the perfectness concept for equilibrium points in extensive games, International Journal of Game Theory, 4 (1975), pp. 25-55.

[27] J. W. Weibull, Evolutionary Game Theory, The MIT Press, 1997. 